\title{
A Short Gamma-ray Burst 'no-host' Problem? Investigating Large Progenitor Offsets for Short Grbs with Optical Afterglows
}

\section{Citation}

Berger, E. 2010. “A SHORT GAMMA-RAY BURST 'NO-HOST' PROBLEM? INVESTIGATING LARGE PROGENITOR OFFSETS FOR SHORT GRBs WITH OPTICAL AFTERGLOWS." The Astrophysical Journal 722 (2): 1946-61. https://doi.org/10.1088/0004-637x/722/2/1946.

\section{Permanent link}

http://nrs.harvard.edu/urn-3:HUL.InstRepos:41397414

\section{Terms of Use}

This article was downloaded from Harvard University's DASH repository, and is made available under the terms and conditions applicable to Other Posted Material, as set forth at http:// nrs.harvard.edu/urn-3:HUL.InstRepos:dash.current.terms-of-use\#LAA

\section{Share Your Story}

The Harvard community has made this article openly available.

Please share how this access benefits you. Submit a story.

Accessibility 


\title{
A SHORT GAMMA-RAY BURST “NO-HOST” PROBLEM? INVESTIGATING LARGE PROGENITOR OFFSETS FOR SHORT GRBs WITH OPTICAL AFTERGLOWS
}

\author{
E. BERGER \\ Harvard-Smithsonian Center for Astrophysics, 60 Garden Street, Cambridge, MA 02138, USA \\ Received 2010 June 29; accepted 2010 August 27; published 2010 October 5
}

\begin{abstract}
We investigate the afterglow properties and large-scale environments of several short-duration gamma-ray bursts (GRBs) with subarcsecond optical afterglow positions but no bright coincident host galaxies. The purpose of this joint study is to robustly assess the possibility of significant offsets, a hallmark of the compact object binary merger model. Five such events exist in the current sample of 20 short bursts with optical afterglows, and we find that their optical, X-ray, and $\gamma$-ray emission are systematically fainter. These differences may be due to lower circumburst densities (by about an order of magnitude), to higher redshifts (by $\Delta z \approx 0.5-1$ ), or to lower energies (by about a factor of 3), although in the standard GRB model the smaller $\gamma$-ray fluences cannot be explained by lower densities. To study the large-scale environments, we use deep optical observations to place limits on underlying hosts and to determine probabilities of chance coincidence for galaxies near each burst. In four of the five cases, the lowest probabilities of chance coincidence $(P(<\delta R) \sim 0.1)$ are associated with bright galaxies at separations of $\delta R \sim 10^{\prime \prime}$, while somewhat higher probabilities of chance coincidence are associated with faint galaxies at separations of $\sim 2^{\prime \prime}$. By measuring redshifts for the brighter galaxies in three cases $(z=0.111,0.473,0.403)$, we find physical offsets of $\approx 30-75 \mathrm{kpc}$, while for the faint hosts the assumption of $z \gtrsim 1$ leads to offsets of $\sim 15 \mathrm{kpc}$. Alternatively, the limits at the burst positions ( $\gtrsim 26 \mathrm{mag}$ ) can be explained by typical short GRB host galaxies $\left(L \approx 0.1-1 L^{*}\right)$ at $z \gtrsim 2-3$. Thus, two possibilities exist: (1) $\sim 1 / 4$ of short GRBs explode $\sim 50 \mathrm{kpc}$ or $\sim 15 \mathrm{kpc}$ from the centers of $z \sim 0.3$ or $z \gtrsim 1$ galaxies, respectively, and have fainter afterglows due to the resulting lower densities; or (2) $\sim 1 / 4$ of short GRBs occur at $z \gtrsim 2$ and have fainter afterglows due to their higher redshifts. The high-redshift scenario leads to a bimodal redshift distribution, with peaks at $z \sim 0.5$ and $z \sim 3$. The large offset scenario leads to an offset distribution that is well matched by theoretical predictions of NS-NS/NS-BH binary kicks, or by a hybrid population with globular cluster NS-NS binaries at large offsets and primordial binaries at offsets of $\lesssim 10 \mathrm{kpc}$ (indicative of negligible kicks). Deeper constraints on any coincident galaxies to $\gtrsim 28 \mathrm{mag}$ (using the Hubble Space Telescope) will allow us to better exclude the high-redshift scenario.
\end{abstract}

Key words: gamma-ray burst: general

Online-only material: color figures

\section{INTRODUCTION}

The bimodality of gamma-ray burst (GRB) durations (Kouveliotou et al. 1993) is indicative of separate progenitor populations for long- and short-duration GRBs. While direct observational support exists for the massive star origin of long GRBs (e.g., Woosley \& Bloom 2006), the most popular progenitor model for short GRBs is the coalescence of compact object binaries with neutron star and/or black hole constituents (NS-NS/NS-BH; Eichler et al. 1989; Paczynski 1991; Narayan et al. 1992). One of the key predictions of this model is that some systems will experience large velocity kicks at birth, leading to eventual mergers well outside of the host galaxies, in galactic halos and the intergalactic medium (IGM; Bloom et al. 1999; Fryer et al. 1999; Belczynski et al. 2006). These models predict that $10 \%-20 \%$ of all mergers will occur at offsets of $\gtrsim 20 \mathrm{kpc}$ for Milky Way mass galaxies. In such environments, the resulting afterglow emission is expected to be fainter than for bursts occurring in coincidence with their host galaxies due to the low ambient density (Panaitescu et al. 2001; Perna \& Belczynski 2002).

A subset of NS-NS binaries $(\sim 10 \%-30 \%)$ may be formed dynamically in globular clusters (Grindlay et al. 2006), leading to possible large offsets and low ambient densities despite an absence of kicks. The distribution of offsets for such binary systems can in principle be calculated from the spatial distribution of globular clusters, and initial predictions are that $60 \%-95 \%$ of these systems will have offsets of $\gtrsim 20 \mathrm{kpc}$ (depending on the mass of the galaxy; Salvaterra et al. 2010). An additional expectation is that dynamically formed binaries will be heavily skewed to lower redshifts due to the additional time delay between the formation and core collapse of the globular clusters (Hopman et al. 2006; Salvaterra et al. 2008).

Other progenitor systems for short GRBs have also been proposed, including young magnetars (Thompson \& Duncan 1995), accretion-induced collapse (AIC) of neutron stars (NSs; Qin et al. 1998), and delayed magnetar formation through binary white dwarf mergers or white dwarf AIC (Levan et al. 2006a; Metzger et al. 2008). These models are partially motivated by observations that cannot be easily accommodated in the standard NS-NS merger model, such as extended soft $\gamma$-ray emission on timescales of $\sim 100$ s (e.g., Villasenor et al. 2005; Metzger et al. 2008; Perley et al. 2009), or by phenomena such as shortduration giant flares from soft $\gamma$-ray repeaters (e.g., Hurley et al. 2005; Palmer et al. 2005; Tanvir et al. 2005; Nakar et al. 2006). The general expectation is that these alternative progenitors will not experience kicks, and will therefore lead to bursts coinciding with host galaxies.

The detection of short GRB afterglows starting in mid-2005 provided an opportunity to investigate the various progenitor models through a range of observational tests: the redshift distribution (Berger et al. 2007; Gal-Yam et al. 2008), the host galaxy demographics (Berger 2009), the afterglow properties (Berger 2007; Gehrels et al. 2008; Kann et al. 2008; Nysewander 
et al. 2009), and perhaps most importantly, their locations relative to the host galaxies (Fong et al. 2010). As of mid-2010, $\mathrm{X}$-ray and optical afterglows have been detected from 40 and 20 short GRBs, respectively, with the latter sample providing accurate subarcsecond positions. Of these 20 events, 15 directly coincide with host galaxies with a wide distribution of apparent magnitudes, and redshifts of $z \approx 0.2-1$ or beyond (e.g., Berger et al. 2007; Berger 2009; D'Avanzo et al. 2009). However, the remaining five events ${ }^{1}$ do not appear to coincide with galaxies, and therefore provide an opportunity to assess the possibility of large progenitor offsets, and to test the validity of the NS-NS merger models.

Significant offsets have been claimed previously, in particular for GRBs 050509b and 060502b with projected offsets of $39 \pm 13$ and $73 \pm 19 \mathrm{kpc}$, respectively (Bloom et al. 2006, 2007). However, in both cases only X-ray positions are available (3".5 and 4." 4 radius, respectively), and the error circles contain several galaxies consistent with a negligible offset (Berger et al. 2007; Bloom et al. 2007). Moreover, in the case of GRB 050509b the X-ray error circle intersected the outer regions of the host, raising the possibility that the progenitor system was formed in, rather than kicked to, the outskirts of the host. This possibility raises a crucial point, namely, that a substantial physical offset from the center of the host does not necessarily point to a progenitor kick if the burst still closely coincides with the host light distribution (Fong et al. 2010). An illustrative example of this point is GRB 071227 whose optical afterglow position coincides with the outskirts of edge-on disk galaxies, with an offset of about $15 \mathrm{kpc}$ from the host center (D'Avanzo et al. 2009; Fong et al. 2010).

Large offsets have also been speculated in a few cases with precise optical afterglow positions (GRBs 061201, 070809, and 080503; Stratta et al. 2007; Perley et al. 2008, 2009). However, these claims have not been investigated systematically, mainly because they were treated on a case-by-case basis, with probabilistic arguments that prevented conclusive associations. These cases, combined with the ambiguity inherent to X-ray positions, demonstrate that bursts with optical afterglows are essential for reaching any robust conclusions about progenitor offsets (due to kicks and/or a globular cluster origin).

Here, we present the first systematic study of short GRBs with optical afterglows and no coincident hosts, which combines their afterglow properties with the large-scale environments. The purpose of this study is to statistically assess the possibility of offsets and to compare this with alternative explanations (e.g., a high-redshift origin). To achieve this goal, we set our study in the broader context of short GRBs that have optical afterglows and coincident hosts, as well as short GRBs with only X-ray positions. As we demonstrate throughout the paper, such a combined study is essential since offsets or high redshifts are expected to jointly affect both the afterglow properties and the large-scale environments. Our study also provides a robust statistical assessment of a posteriori chance coincidence probabilities, and the expected number of spurious associations, which cannot be properly assessed for individual bursts.

The paper is organized as follows. In Section 2, we present deep optical observations of the environments of GRBs 061201, 070809, 080503, 090305, and 090515, as well as spectroscopic observations of bright galaxies near the burst positions for GRBs

\footnotetext{
1 These are GRBs 061201: Berger (2006a), Stratta et al. (2007), and Fong et al. (2010); 070809: Perley et al. (2007, 2008); 080503: Perley et al. (2009); 090305: Cenko et al. (2009) and Berger \& Kelson (2009); and 090515: Rowlinson et al. (2010b).
}

061201, 070809, and 090515. We study trends in the afterglow and prompt emission properties of bursts with and without coincident hosts in Section 3, and determine a posteriori probabilities of chance coincidence as a function of projected angular offset for galaxies near the position of each burst in Section 4. We also determine projected physical and host-normalized offsets, and use these in conjunction with the afterglow and prompt emission properties to address two scenarios for the short bursts with optical emission and no coincident bright hosts: (1) an origin in faint galaxies at $z \gtrsim 2$ (Section 4.2), or (2) substantial offsets from galaxies at $z \sim 0.1-0.5$ or $z \gtrsim 1$ (Section 4.3). Finally, in Section 5 we present the offset distribution of all short GRBs with optical afterglows (in the context of scenario (2)), and compare this distribution with predictions for NS-NS kicks and dynamically formed NS-NS binaries. We further investigate whether the circumburst densities that are required for the measured optical magnitudes can be accommodated with a halo or IGM origin. We draw conclusions about the progenitors of short GRBs from our systematic study in Section 6.

\section{SHORT GRB SAMPLE AND OBSERVATIONS}

We include in this investigation all 20 short GRBs with optical afterglow detections as of 2010 June. This is the full subset of events for which subarcsecond positions are available. We stress that this sample represents only about $1 / 3$ of all short GRBs discovered to date, and about $1 / 2$ of the sample with X-ray afterglow detections. Thus, it is not a complete sample of short GRBs, but it is the only subset for which we can investigate the possibility of large offsets with meaningful statistical significance. As we demonstrate in Section 5, we do not expect this sample to be strongly biased with respect to circumburst density, at least for $n \gtrsim 10^{-5} \mathrm{~cm}^{-3}$. The properties of the 20 short GRBs, as well as events with deep optical limits, are summarized in Table 1. As can be inferred from the table, some of the 20 events with only X-ray detections do not have optical follow-up observations, suggesting that the sample with optical afterglows may be largely representative.

For the purpose of our investigation, we define three subsamples that will be used throughout the paper: (1) Sample 1: short GRBs with detected afterglows and coincident host galaxies (15 bursts), (2) Sample 2: the five short bursts with detected optical afterglows and no bright coincident hosts, and (3) Sample 3: short GRBs with detected X-ray afterglows (from the Swift X-ray Telescope (XRT)) but no optical detections despite rapid follow-up observations (13 bursts).

\subsection{Optical Imaging}

For the bursts in Sample 2, we use deep space- and groundbased optical observations to place limits on the brightness of underlying galaxies, and to assess the probability of chance coincidence for nearby galaxies. For GRB 061201, we use Hubble Space Telescope (HST) observations obtained with the Advanced Camera for Surveys (ACS) in the F814W filter, with a total exposure time of $2244 \mathrm{~s}$ (Fong et al. 2010). For GRB 070809, we use $r$-band observations obtained on 2008 January 14 UT with the Low Dispersion Survey Spectrograph (LDSS3) mounted on the Magellan/Clay $6.5 \mathrm{~m}$ telescope, with a total exposure time of $1500 \mathrm{~s}$. For GRB 080503, we use HST Wide-Field Planetary Camera 2 (WFPC2) observations in the F606W filter from 2009 January 30 UT, with a total exposure time of $4000 \mathrm{~s}$ (Perley et al. 2009). We also use the limits on a coincident host inferred from a deeper stack of HST observations 
Table 1

Properties of Short GRBs with Optical Afterglows or Limits

\begin{tabular}{|c|c|c|c|c|c|c|c|c|}
\hline GRB & $\begin{array}{l}T_{90} \\
(\mathrm{~s})\end{array}$ & $z^{\mathrm{a}}$ & $\begin{array}{c}F_{\gamma}^{\mathrm{b}} \\
\left(\operatorname{erg~cm}^{-2}\right) \\
\end{array}$ & $\begin{array}{c}t_{X} \\
(\mathrm{hr}) \\
\end{array}$ & $\begin{array}{c}F_{X}^{\mathrm{c}} \\
\left(\mathrm{erg} \mathrm{cm}^{-2} \mathrm{~s}^{-1}\right)\end{array}$ & $\begin{array}{l}t_{\mathrm{opt}} \\
(\mathrm{hr})\end{array}$ & $\begin{array}{l}F_{v, \mathrm{opt}} \\
(\mu \mathrm{Jy})\end{array}$ & Refs. \\
\hline \multicolumn{9}{|c|}{ Short GRBs with optical detections (Samples 1 and 2) } \\
\hline 050709 & 0.07 & 0.161 & $2.9 \times 10^{-7}$ & 60.5 & $3.5 \times 10^{-15}$ & 34 & 2.3 & $1-3$ \\
\hline 050724 & 3.0 & 0.257 & $3.9 \times 10^{-7}$ & 11.8 & $1.7 \times 10^{-12}$ & 12 & 8.4 & $4-6$ \\
\hline $051221 \mathrm{~A}$ & 1.4 & 0.546 & $1.2 \times 10^{-6}$ & 3.1 & $1.8 \times 10^{-12}$ & 3.1 & 5.8 & $7-8$ \\
\hline 060121 & 2.0 & $<4.3$ & $4.7 \times 10^{-6}$ & 6.1 & $1.8 \times 10^{-12}$ & 7.4 & 8.8 & $9-11$ \\
\hline 060313 & 0.70 & $<3$ & $1.1 \times 10^{-6}$ & 2.8 & $4.0 \times 10^{-12}$ & 2.8 & 10.8 & $11-12$ \\
\hline 061006 & 0.42 & 0.438 & $1.4 \times 10^{-6}$ & 19.5 & $1.0 \times 10^{-13}$ & 14.9 & 2.9 & 11,13 \\
\hline $061201^{\mathrm{d}}$ & 0.80 & $<1.7,0.111 ?$ & $3.3 \times 10^{-7}$ & 8.6 & $2.5 \times 10^{-13}$ & 8.6 & 2.9 & $14-15$ \\
\hline 070707 & 1.1 & $<4.3$ & $1.4 \times 10^{-6}$ & 12.5 & $2.3 \times 10^{-13}$ & 11 & 1.9 & 16 \\
\hline 070714B & 3.0 & 0.923 & $7.2 \times 10^{-7}$ & 24.4 & $1.5 \times 10^{-14}$ & 23.6 & 0.7 & 17 \\
\hline 070724 & 0.40 & 0.457 & $3.0 \times 10^{-8}$ & 2.35 & $5.5 \times 10^{-13}$ & 2.3 & 5.0 & $18-19$ \\
\hline $070809^{d}$ & 1.3 & $<3,0.473 ?$ & $1.0 \times 10^{-7}$ & 11.0 & $3.0 \times 10^{-13}$ & 11 & 0.8 & $20-21$ \\
\hline 071227 & 1.8 & 0.381 & $2.2 \times 10^{-7}$ & 7.0 & $6.9 \times 10^{-14}$ & 7.0 & 1.6 & 13 \\
\hline $080503^{d}$ & 0.32 & $<3, \quad \cdots$ & $6.1 \times 10^{-8}$ & 66.0 & $<7.8 \times 10^{-14}$ & 25.9 & 0.3 & 22 \\
\hline 080905 & 1.0 & 0.122 & $1.4 \times 10^{-7}$ & 18.4 & $<5.9 \times 10^{-14}$ & 8.5 & 0.8 & 23 \\
\hline $090305^{d}$ & 0.40 & $<4.3, \cdots$ & $7.5 \times 10^{-8}$ & 0.45 & $<8.5 \times 10^{-14}$ & 0.45 & 1.5 & $24-25$ \\
\hline 090426 & 1.28 & 2.609 & $2.5 \times 10^{-7}$ & 2.0 & $1.1 \times 10^{-12}$ & 2.6 & 20.0 & $26-27$ \\
\hline 090510 & 0.30 & 0.903 & $3.4 \times 10^{-7}$ & 6.4 & $1.7 \times 10^{-13}$ & 9.0 & 2.3 & 28 \\
\hline $090515^{d}$ & 0.04 & $<4.3,0.403 ?$ & $2.1 \times 10^{-8}$ & 4.9 & $<9.2 \times 10^{-14}$ & 1.9 & 0.1 & 29 \\
\hline 091109B & 0.30 & $\ldots$ & $1.9 \times 10^{-7}$ & 3.8 & $1.9 \times 10^{-13}$ & 5.7 & 0.5 & 30 \\
\hline 100117 & 0.30 & 0.92 & $9.3 \times 10^{-8}$ & 8.4 & $<2.0 \times 10^{-14}$ & 8.4 & 0.3 & 31 \\
\hline \multicolumn{9}{|c|}{ Short GRBs with optical limits (Sample 3) } \\
\hline 050509B & 0.04 & 0.225 & $9.5 \times 10^{-9}$ & 9.1 & $<2.0 \times 10^{-14}$ & 2.1 & $<0.7$ & $32-33$ \\
\hline 050813 & 0.60 & $\cdots$ & $1.2 \times 10^{-7}$ & 94.9 & $<2.6 \times 10^{-14}$ & 12.8 & $<1.9$ & $34-36$ \\
\hline 051210 & 1.27 & $\ldots$ & $8.1 \times 10^{-8}$ & 6.9 & $<2.9 \times 10^{-14}$ & 19.2 & $<1.6$ & 11,37 \\
\hline 060502B & 0.09 & $\cdots$ & $4.0 \times 10^{-8}$ & 18.6 & $<9.1 \times 10^{-14}$ & 16.8 & $<0.7$ & 11,38 \\
\hline 060801 & 0.50 & 1.130 & $8.1 \times 10^{-8}$ & 10.9 & $<9.0 \times 10^{-15}$ & 12.4 & $<0.8$ & 11 \\
\hline 061210 & 0.19 & 0.409 & $1.1 \times 10^{-6}$ & 72.4 & $<6.7 \times 10^{-14}$ & 2.1 & $<1.4$ & 11 \\
\hline 061217 & 0.21 & 0.827 & $4.6 \times 10^{-8}$ & 38.6 & $<1.7 \times 10^{-14}$ & 2.8 & $<2.0$ & 11 \\
\hline 070429B & 0.50 & 0.902 & $6.3 \times 10^{-8}$ & 11.8 & $<3.8 \times 10^{-14}$ & 4.8 & $<0.6$ & 39 \\
\hline 080426 & 1.30 & $\ldots$ & $3.7 \times 10^{-7}$ & 6.1 & $2.9 \times 10^{-13}$ & 7.5 & $<2.6$ & 40 \\
\hline 080702 & 0.50 & $\cdots$ & $3.6 \times 10^{-8}$ & 2.9 & $<4.0 \times 10^{-14}$ & 12.1 & $<12.3$ & 41 \\
\hline 081226 & 0.40 & $\ldots$ & $9.9 \times 10^{-8}$ & 3.2 & $<2.9 \times 10^{-14}$ & 1.6 & $<0.9$ & 42 \\
\hline 090621B & 0.14 & $\cdots$ & $7.0 \times 10^{-8}$ & 1.9 & $1.4 \times 10^{-14}$ & 0.9 & $<3.3$ & 43 \\
\hline 100206 & 0.12 & $\cdots$ & $1.4 \times 10^{-7}$ & 6.9 & $<2.0 \times 10^{-14}$ & 15.7 & $<0.5$ & 44 \\
\hline
\end{tabular}

Notes. Prompt emission and afterglow data for short GRBs with detected optical afterglows (top section) and deep optical afterglow limits (bottom section).

${ }^{a}$ Redshifts include spectroscopic measurements, limits from afterglow detections in the UV/optical, and for the bursts in Sample 2, redshifts for galaxies with the lowest probability of chance coincidence (marked by “?”).

$\mathrm{b}$ The fluences are in the observed 15-150 keV band, with the exception of GRB 050709 (2-400 keV) and GRB 060121 (2-400 keV).

c All XRT data are from Evans et al. (2007) and Evans et al. (2009).

d Short GRBs in Sample 2.

References. (1) Villasenor et al. 2005; (2) Fox et al. 2005; (3) Hjorth et al. 2005; (4) Barthelmy et al. 2005; (5) Berger et al. 2005; (6) Grupe et al. 2006; (7) Burrows et al. 2006; (8) Soderberg et al. 2006; (9) de Ugarte Postigo et al. 2006; (10) Levan et al. 2006b; (11) Berger et al. 2007; (12) Roming et al. 2006; (13) D’Avanzo et al. 2009; (14) Stratta et al. 2007; (15) Fong et al. 2010; (16) Piranomonte et al. 2008; (17) Graham et al. 2009; (18) Berger et al. 2009; (19) Kocevski et al. 2010; (20) Perley et al. 2007; (21) Perley et al. 2008; (22) Perley et al. 2009; (23) Rowlinson et al. 2010a; (24) Cenko et al. 2009; (25) Berger \& Kelson 2009; (26) Antonelli et al. 2009; (27) Levesque et al. 2010; (28) McBreen et al. 2010; (29) Rowlinson et al. 2010b; (30) Malesani et al. 2009; (31) W. Fong et al. 2011, in preparation; (32) Gehrels et al. 2005; (33) Bloom et al. 2006; (34) Ferrero et al. 2007; (35) Berger 2006b; (36) Prochaska et al. 2006; (37) La Parola et al. 2006; (38) Bloom et al. 2007; (39) Cenko et al. 2008; (40) de Ugarte Postigo et al. 2008; (41) Greco et al. 2008; (42) Berger et al. 2008; (43) Galeev et al. 2009; (44) Berger et al. 2010.

by Perley et al. (2009), with $m_{\mathrm{AB}}(\mathrm{F} 606 \mathrm{~W}) \gtrsim 28.5 \mathrm{mag}$. For GRB 090305, we use LDSS3 $r$-band observations obtained on 2010 May 8 UT with a total exposure time of 2400 s. Finally, for GRB 090515 we use $r$-band observations obtained with the Gemini Multi-Object Spectrograph (GMOS) mounted on the Gemini-North $8 \mathrm{~m}$ telescope from 2009 May 15 UT with a total exposure time of $1800 \mathrm{~s}$.

The ground-based observations were reduced and analyzed using standard routines in IRAF. The analysis of the HST observations is detailed in Fong et al. (2010). The limiting magnitudes for all five observations are listed in Table 2, and images of the five fields are shown in Figures 1-5.

\subsection{Optical Spectroscopy}

In addition to the imaging observations, we obtained spectroscopic observations of galaxies near the positions of GRBs 061201, 070809, and 090515. Our observations of a galaxy 


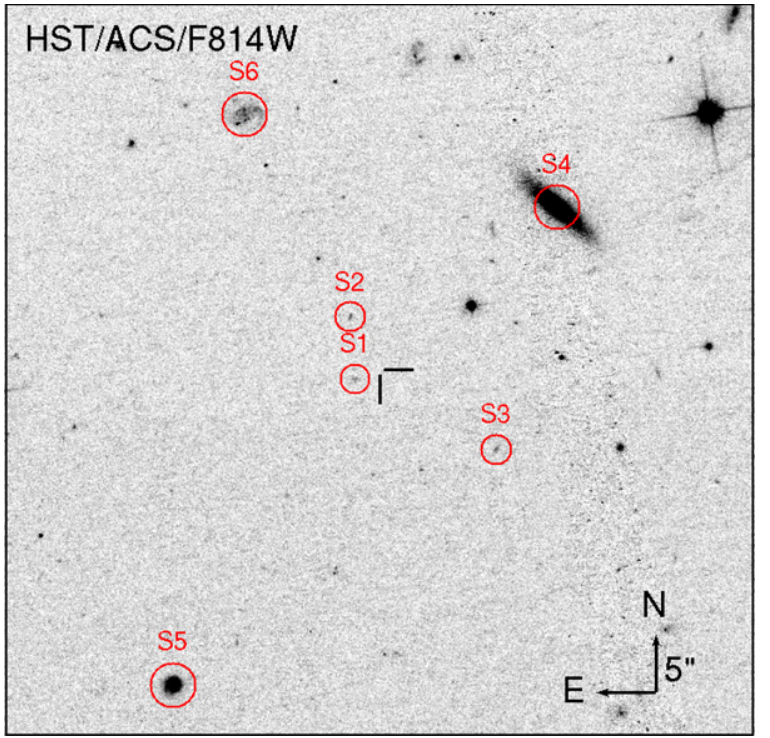

Figure 1. HST/ACS/F814W image of the location of GRB 061201. Galaxies near the position of the optical afterglow (cross-hairs) are marked. (A color version of this figure is available in the online journal.)

located 16".3 from the afterglow position of GRB 061201 (marked "S4" in Figure 1) revealed a star-forming galaxy at $z=0.111$ (Stratta et al. 2007; Fong et al. 2010); see Figure 6 .

For GRB 070809, we obtained spectra of two galaxies located 5.9 and 6".0 from the optical afterglow position (marked "S2" and "S3," respectively, in Figure 2) using LDSS3 on 2008 January 14 UT. The galaxy at a separation of 5".9 was previously identified as a star-forming galaxy at $z=0.218$ by Perley et al. (2008). Here, we find that the object at a separation of 6"' 0 is an early-type galaxy at $z=0.473$, with no evidence for ongoing star formation activity (Figure 7).

Finally, for GRB 090515 we obtained multi-object spectroscopic observations with LDSS3 for nearly 100 galaxies within a $5^{\prime} \times 5^{\prime}$ field centered on the GRB position. These observations provide redshifts for several galaxies near the host position, including a star-forming galaxy at $z=0.626$ (5".8 offset; "S1" in Figure 5), an early-type galaxy at $z=0.403$ (14".0 offset; "S5"), and a star-forming galaxy at $z=0.657$ (14".9 offset; "S6"); see
Table 2

Observations of Short GRBs with Optical Afterglows and no Coincident Host Galaxies (Sample 2)

\begin{tabular}{ccccc}
\hline \hline GRB & Instrument & Filter & $\begin{array}{c}t_{\exp } \\
(\mathrm{s})\end{array}$ & $\begin{array}{c}m_{\mathrm{lim}}{ }^{\mathrm{a}} \\
(\mathrm{AB} \mathrm{mag})\end{array}$ \\
\hline 061201 & HST/ACS & F814W & 2224 & 26.0 \\
070809 & Magellan/LDSS3 & $r$ & 1500 & 25.4 \\
080503 & HST/WFPC2 & F606W & 4000 & $28.5^{\mathrm{b}}$ \\
090305 & Magellan/LDSS3 & $r$ & 2400 & 25.6 \\
090515 & Gemini-N/GMOS & $r$ & 1800 & 26.5 \\
\hline
\end{tabular}

Notes.

${ }^{\text {a }}$ Limits are $3 \sigma$.

b This limit is from Perley et al. (2009).

Figure 8. In an upcoming paper, we will demonstrate that the galaxy at $z=0.403$ is a member of a cluster.

\section{AFTERGLOW PROPERTIES}

We begin our investigation by assessing the distribution of optical afterglow magnitudes for the three samples defined in Section 2. The observed magnitudes and limits as a function of time after the burst are shown in Figure 9. The median observation time for the sample is about $8.5 \mathrm{hr}$ after the burst. The distribution of detected optical afterglow magnitudes is broad, ranging from $r_{\mathrm{AB}} \approx 21$ to $\approx 26 \mathrm{mag}$. The limits range from $r_{\mathrm{AB}} \gtrsim 23$ to $\gtrsim 25 \mathrm{mag}$ (with the exception of GRB 080702 which only has a shallow limit of about $21 \mathrm{AB}$ mag; Greco et al. 2008).

The mean brightness and standard deviation for Sample 1 are $^{2}$ $\left\langle r_{\mathrm{AB}}\right\rangle=23.0 \pm 1.3 \mathrm{mag}$. This is substantially fainter, by about an order of magnitude, than the afterglows of long GRBs on a comparable timescale (e.g., Kann et al. 2008, 2010). It is also remarkably similar to the prediction of $R \approx 23 \mathrm{mag}$ at $t \approx 10 \mathrm{hr}$ by Panaitescu et al. (2001). Since the available limits are at least $\gtrsim 23 \mathrm{mag}$, we conclude that the bursts lacking optical detections are drawn from a population with fainter afterglows. For the five bursts in Sample 2, we find a median and standard deviation of

\footnotetext{
2 These numbers remain essentially unchanged if we extrapolate all measured magnitudes to the fiducial time of $8.5 \mathrm{hr}$ with a typical afterglow decay index of $\alpha=-0.75$.
}
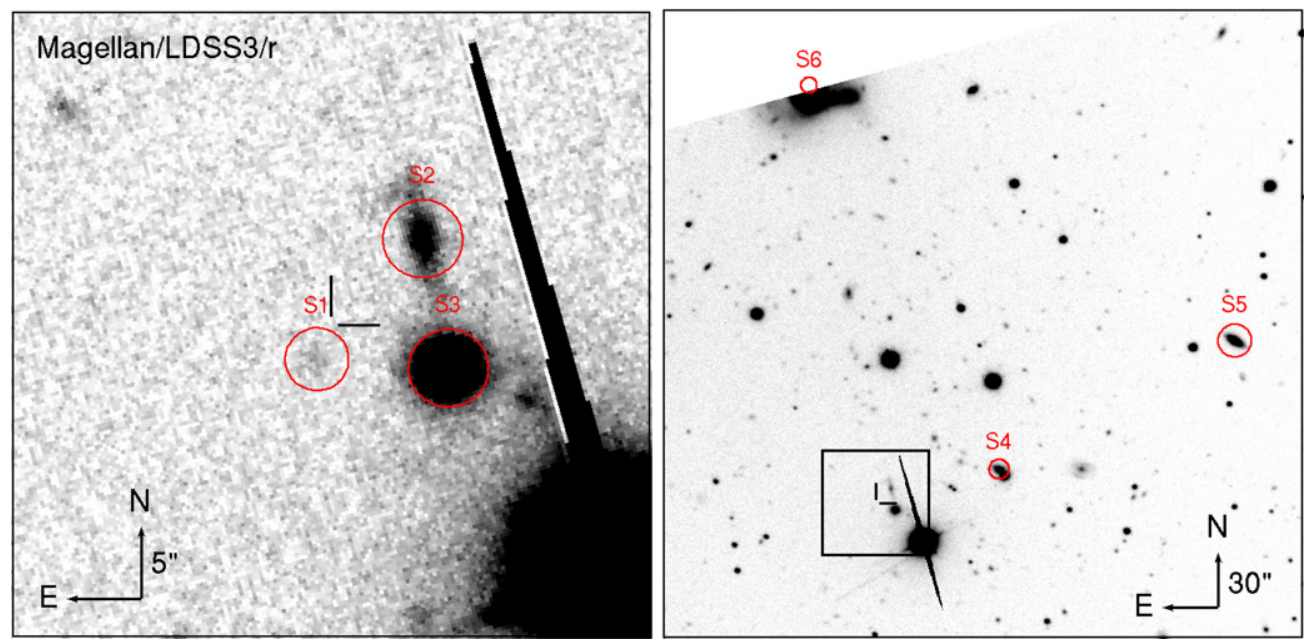

Figure 2. Magellan/LDSS3 $r$-band images of the location of GRB 070809. Galaxies near the position of the optical afterglow (cross-hairs) are marked. (A color version of this figure is available in the online journal.) 


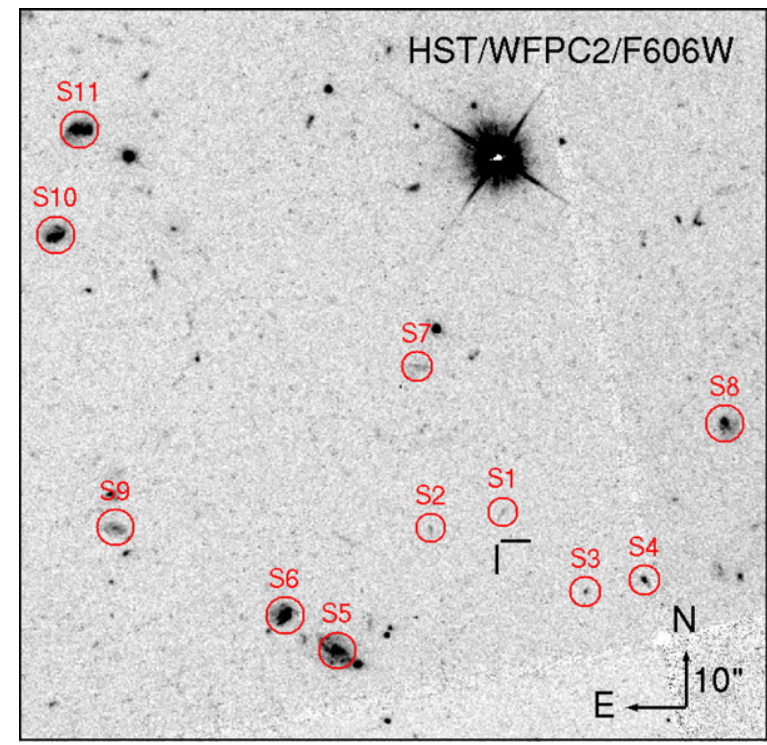

Figure 3. $H S T$ /WFPC2/F606W image of the location of GRB 080503. Galaxies near the position of the optical afterglow (cross-hairs) are marked. A faint galaxy at a separation of only $00^{\prime \prime} 8$ was found by Perley et al. (2009) based on a deeper stack of $H S T$ /WFPC2 observations. These authors also find that the galaxy marked "S5" is located at $z=0.561$, leading to a physical offset of $85 \mathrm{kpc}$.

(A color version of this figure is available in the online journal.)

$\left\langle r_{\mathrm{AB}}\right\rangle=24.4 \pm 1.4 \mathrm{mag}$, about 1.4 mag fainter than the bursts with coincident hosts.

The cumulative distributions for the three samples normalized to the fiducial time of $8.5 \mathrm{hr}$ after the burst are shown in Figure 10. A Kolmogorov-Smirnov (K-S) test indicates that there is only an $8 \%$ probability that Sample 1 and Sample 2 are drawn from the same underlying distribution of optical afterglow brightnesses. Similarly, the probability that Sample 1 and Sample 3 are drawn from the same underlying distribution is $\lesssim 5 \%$ (an upper limit since the bursts in Sample 3 are not detected in the optical). On the other hand, the probability that Sample 2 and Sample 3 are drawn from the same underlying distribution is high, $\approx 50 \%$.

The overall faintness of the optical afterglows in Sample 2 and Sample 3 can be explained in two primary ways. First, they could result from systematically lower circumburst densities. In the standard afterglow model ${ }^{3}$ with $v_{m}<v_{\mathrm{opt}}<v_{c}$, the afterglow flux scales as $F_{v} \propto n^{1 / 2}$ for a uniform medium (Sari et al. 1998). Thus, a difference of about +1.4 mag can be explained with a density that is about an order of magnitude lower than for the bursts in Sample 1.

Alternatively, the fainter fluxes may be due to higher redshifts for Sample 2 and Sample 3 compared to Sample 1 since the optical flux also scales as $F_{v} \propto(1+z)^{(3+p) / 4} d_{L}^{-2}$, where $d_{L}$ is the luminosity distance. The +1.4 mag difference corresponds to $\Delta z \approx+0.5(+1)$ for a Sample 1 mean redshift of $z=0.5$ $(z=1)$. Similarly, the flux also depends on the total energy, with $F_{v} \propto E^{(3+p) / 4}$, and therefore a difference of +1.4 mag can be explained with an energy release lower by about a factor of 3.

The various scenarios (lower density, lower energy, or higher redshift) can be further explored through a comparison of the prompt $\gamma$-ray emission, and the relation between the optical and $\mathrm{X}$-ray afterglow brightness. In the framework of the standard GRB model, we do not expect lower densities to impact the prompt emission since it is expected to be produced by internal processes (shocks or magnetic dissipation) that do not depend on the external medium. On the other hand, lower energies or higher redshifts will tend to systematically affect the prompt and afterglow emission.

In Figure 11, we plot the distributions of $\gamma$-ray fluence $\left(F_{\gamma}\right)$, afterglow X-ray flux at the fiducial time of $8 \mathrm{hr}\left(F_{X, 8}\right)$, and duration $\left(T_{90}\right)$ for the three samples. For the latter, we only use the duration of the short component when extended soft emission is also detected. This provides a comparison of the key feature that designates the bursts as short. The distributions allow us to explore the underlying reason for the difference in optical afterglow brightness between the three samples. First, we find that the distribution of the $F_{\gamma}$ values for Sample 1 has a mean value that is about a factor of 5 times larger than for Sample 2 and Sample 3. This is indicative of higher redshifts for the latter two samples if the isotropic-equivalent energies of all short GRBs are similar, or alternatively a lower energy scale if the redshifts are similar.

\footnotetext{
3 We use the standard synchrotron spectrum definitions: $v_{m}$ is the characteristic synchrotron frequency corresponding to electrons with the minimum Lorentz factor $\left(\gamma_{m}\right)$ of the electron distribution, $N(\gamma) \propto \gamma^{-p} ; p$ usually has a value of $\approx 2.2-2.5$; and $v_{c}$ is the synchrotron cooling frequency (Sari et al. 1998).
}
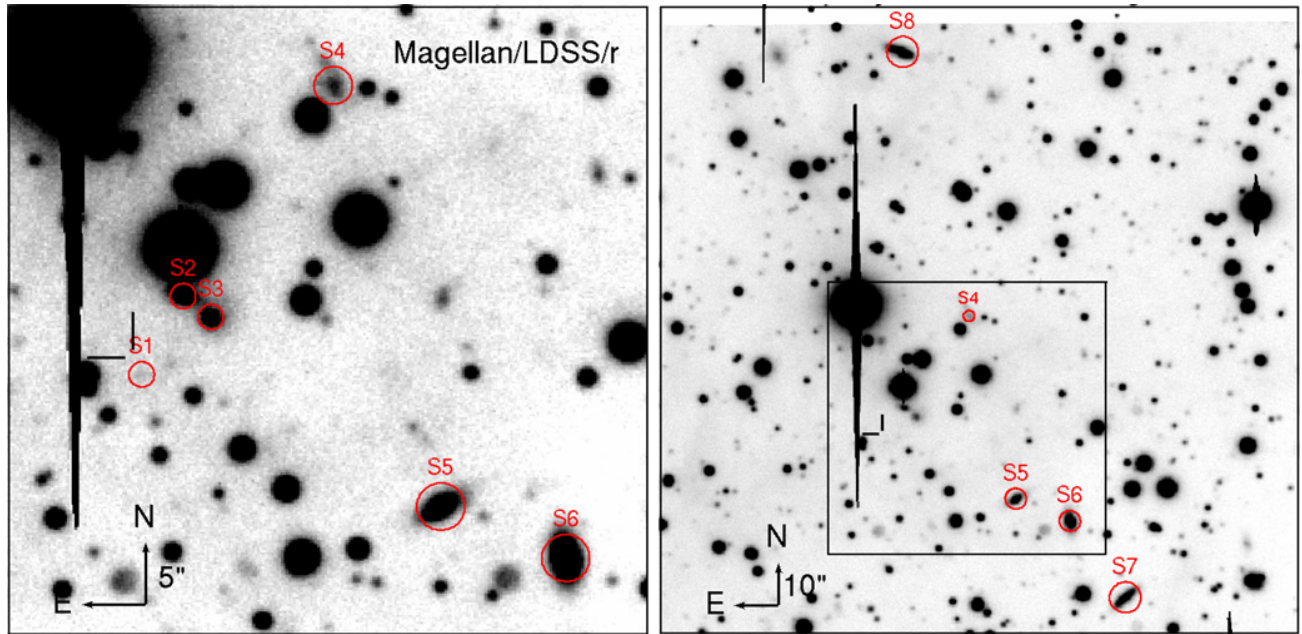

Figure 4. Magellan/LDSS3 $r$-band images of the location of GRB 090305. Galaxies near the position of the optical afterglow (cross-hairs) are marked. (A color version of this figure is available in the online journal.) 

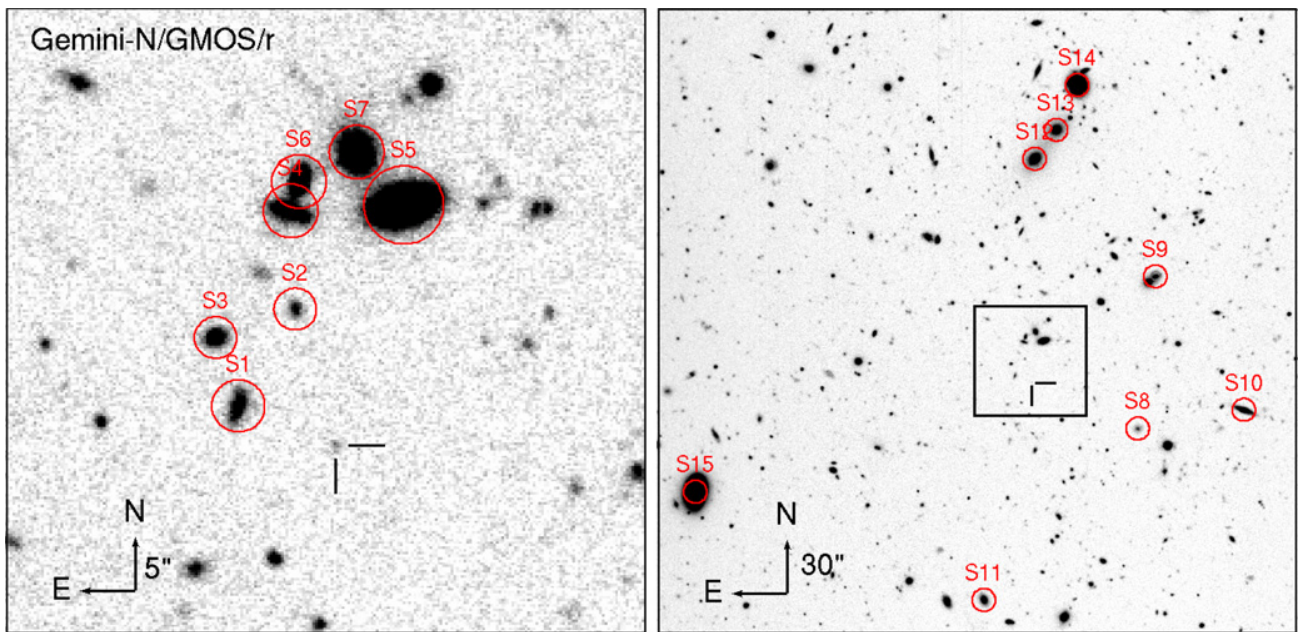

Figure 5. Gemini-North/GMOS $r$-band images of the location of GRB 090515. Galaxies near the position of the optical afterglow (cross-hairs) are marked. Note that the object coincident with the cross-hairs is the optical afterglow.

(A color version of this figure is available in the online journal.)

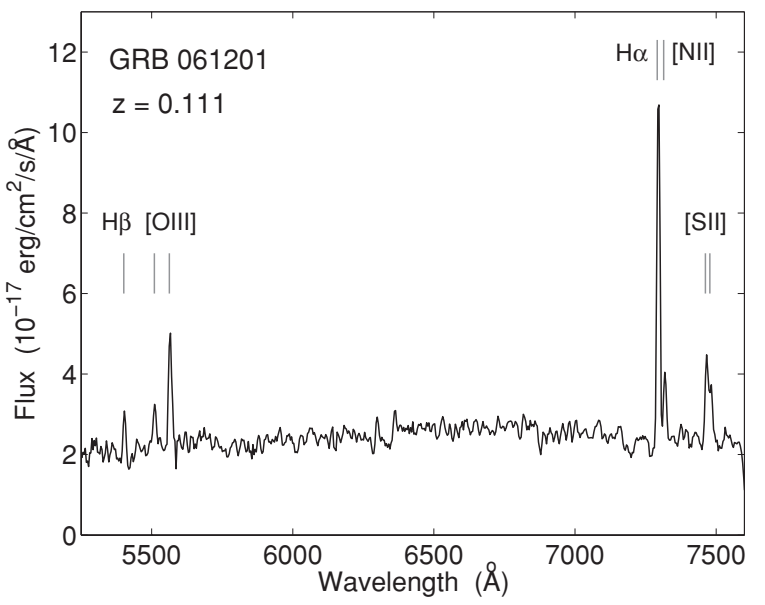

Figure 6. Magellan/LDSS3 spectrum of the galaxy with the lowest probability of chance coincidence near the position of GRB 061201. This galaxy is marked "S4" in Figure 1. It has a redshift of $z=0.111$ and it is undergoing active star formation (Berger 2006a; Stratta et al. 2007; Fong et al. 2010).

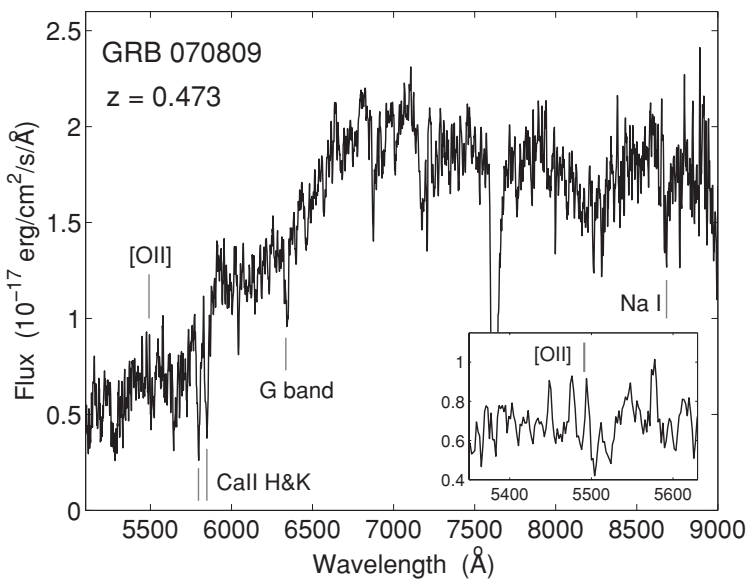

Figure 7. Magellan/LDSS3 spectrum of the galaxy with the lowest probability of chance coincidence near the position of GRB 070809. This galaxy is marked "S3" in Figure 2. It has a redshift of $z=0.473$ and is an early-type galaxy with no evidence for ongoing star formation activity (see inset).

In the same vein, we find that the distributions of the $F_{X, 8}$ values for Sample 2 and Sample 3 have lower means than for

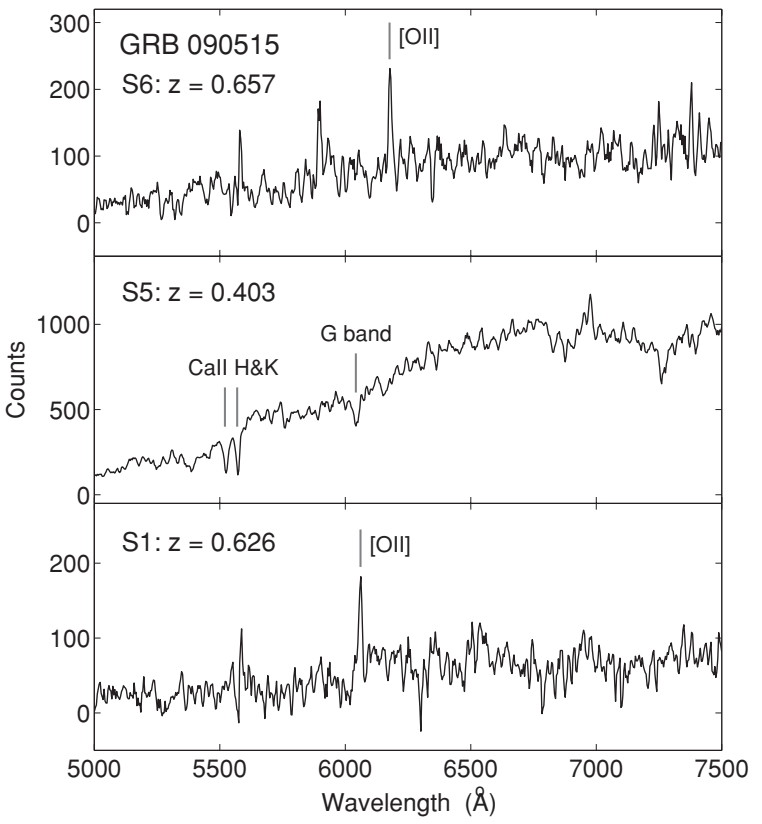

Figure 8. Magellan/LDSS3 spectra of three galaxies with a low probability of chance coincidence near the position of GRB 090515. The galaxy with the lowest probability of chance coincidence is marked "S5" in Figure 5. It has a redshift of $z=0.403$ and is an early-type galaxy which is part of a galaxy cluster (W. Fong et al. 2011, in preparation).

Sample 1. This result is indicative of overall fainter afterglow emission for the former two samples, and this can again be explained in the context of lower energies or higher redshifts. Unlike in the case of the $\gamma$-ray emission, a lower circumburst density would also account for the fainter X-ray fluxes if $v_{X}<v_{c}$. Finally, we find that the durations of the bursts in Sample 2 and Sample 3 are shorter by about a factor of 2 compared to the events with optical afterglows and coincident hosts, although there is substantial scatter in all three samples. The shorter durations are not trivially explained in the context of lower energies, higher redshifts, or lower densities.

To explore this result in more detail, we plot the observed fluence as a function of duration for the events in all three samples (Figure 12). We find that there is a mild positive 


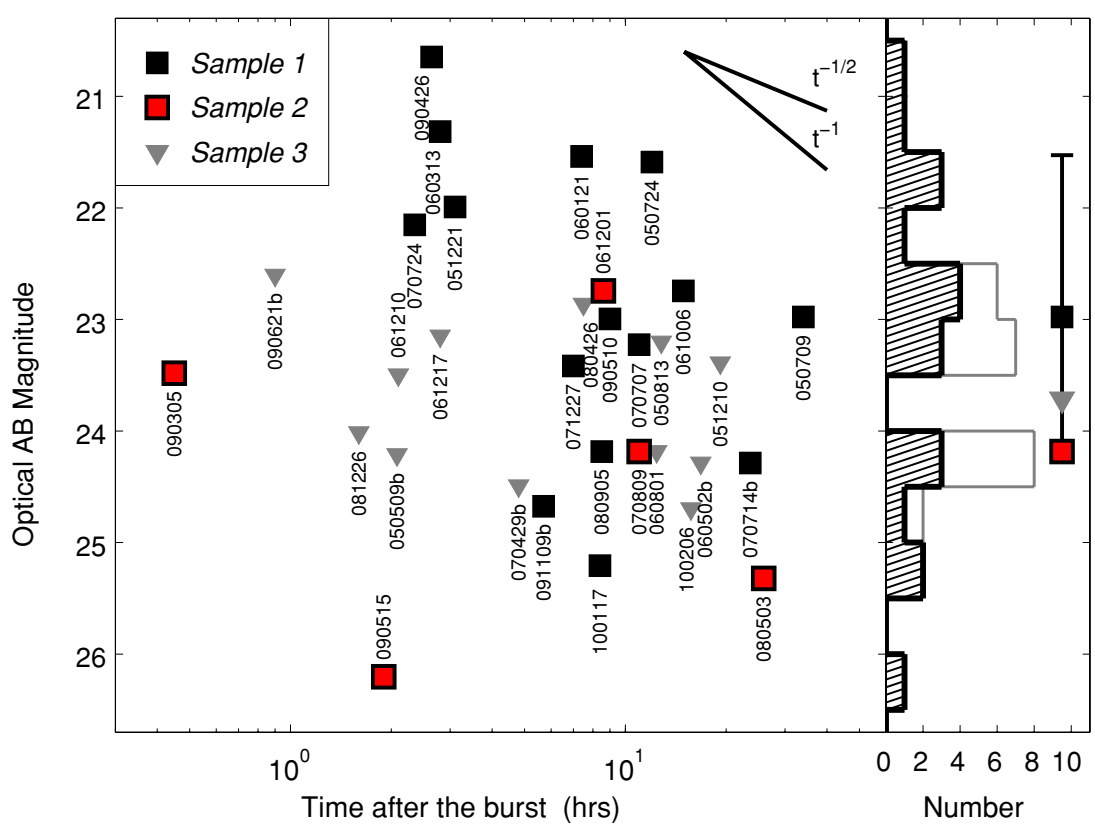

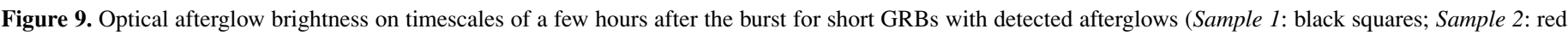

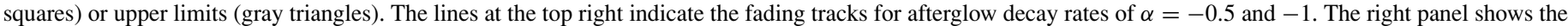

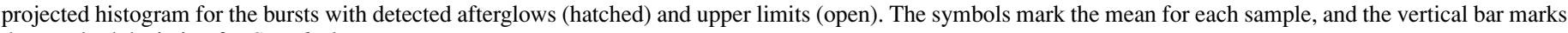
the standard deviation for Sample 1.

(A color version of this figure is available in the online journal.)

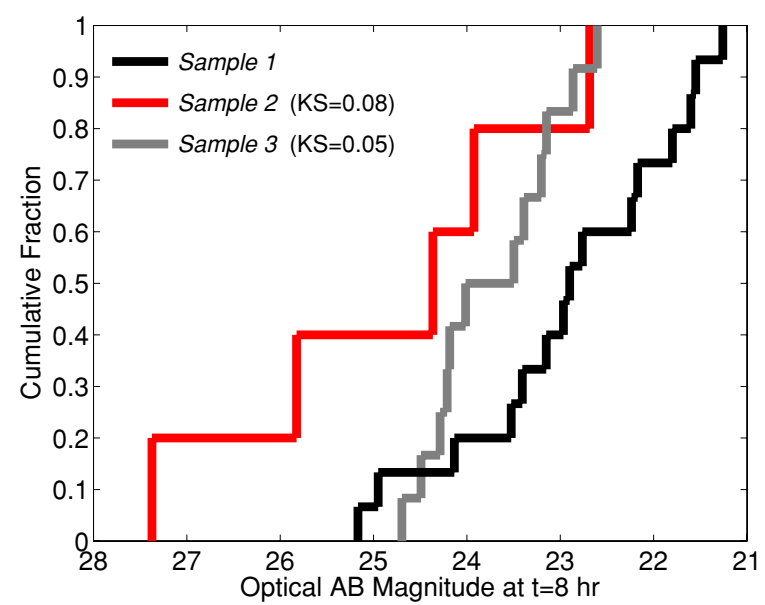

Figure 10. Cumulative afterglow brightness distributions for the bursts in Figure 9, extrapolated to a common fiducial time of $8 \mathrm{hr}$ after the burst with a fading rate of $\alpha=-0.75$. The K-S probabilities relative to the sample with detected afterglows and coincident hosts are noted in the figure. It appears unlikely that the bursts with no coincident hosts, and those with deep upper limits, are drawn from the same distribution as the bursts with detected hosts.

(A color version of this figure is available in the online journal.)

correlation between the two quantities, but that the events in Sample 1 appear to have larger fluences at a given duration compared to the events in Sample 2 and Sample 3. This is indicative of lower $\gamma$-ray fluxes for the latter two samples, possibly as a result of higher redshifts. Higher redshifts will also shift the intrinsic durations of the bursts in Sample 2 and Sample 3 into better agreement with the bursts in Sample 1.

We therefore conclude that the differences in prompt emission and optical/X-ray afterglow properties are consistent with a higher redshift origin for the bursts in Sample 2 and Sample 3 . The fainter afterglow emission is also consistent with lower density environments for these two samples, although this does not clearly explain the differences in prompt emission (at least in the framework of the standard GRB model). We return to the discussion of low density versus a high-redshift origin in Section 4 when we investigate the host galaxy properties.

In addition to the overall faintness of the optical and $\mathrm{X}$-ray afterglows, a substantial difference in density may also be imprinted on the ratio of optical to X-ray brightness. This is because the synchrotron cooling frequency depends on density as $v_{c} \propto n^{-1}$, and is therefore expected to transition across the X-ray band as the density decreases. For $v_{c}>v_{X}$, the X-ray and optical bands occupy the same portion of the synchrotron spectrum, with a resulting spectral index of $\beta=-(p-1) / 2 \approx-0.6$ to $\approx-0.75$ while for $v_{c}<v_{X}$ (i.e., high density), the spectrum between the two bands will be steeper, reaching a maximum value of $\approx-1.25$ when $v_{c} \approx v_{\text {opt }}$. In Figure 13 , we plot the X-ray flux versus optical magnitude for all three samples. For each burst, the fluxes in the optical and X-rays are taken at similar times after the burst, or extrapolated to a common time. The correction factors due these extrapolations are generally ${ }^{4} \lesssim 2$ (Table 1). For the combined Sample 1 and Sample 2, we find a clear correlation between the fluxes in the two bands, leading to a mean optical to X-ray spectral index of $\left\langle\beta_{\mathrm{OX}}\right\rangle=-0.72 \pm 0.17$. This is essentially indistinguishable from the ratio for long GRBs, $\left\langle\beta_{\mathrm{OX}}\right\rangle=-0.65 \pm 0.35$ (Jakobsson et al. 2004). The median values are consistent with $v_{c} \gtrsim v_{X}$, but exhibit dispersion that is likely due to scatter in the values of $p$ and/or the location of $v_{c}$ relative to the $\mathrm{X}$-ray band.

The similarity of $\beta_{\mathrm{OX}}$ for long and short GRBs does not necessarily indicate that the densities are similar for the two samples. In particular, if $v_{c}$ is located close to the X-ray band for long GRBs, while for short GRBs $v_{c} \gg v_{\mathrm{X}}$ (due to a lower density), the effect on $\beta_{\text {OX }}$ will be marginal, particularly within the overall observed scatter. For example, with $p=2.5$ the difference in $\beta_{\mathrm{OX}}$ between a model with $v_{c}$ exactly intermediate

\footnotetext{
4 We do not extrapolate X-ray upper limits.
} 

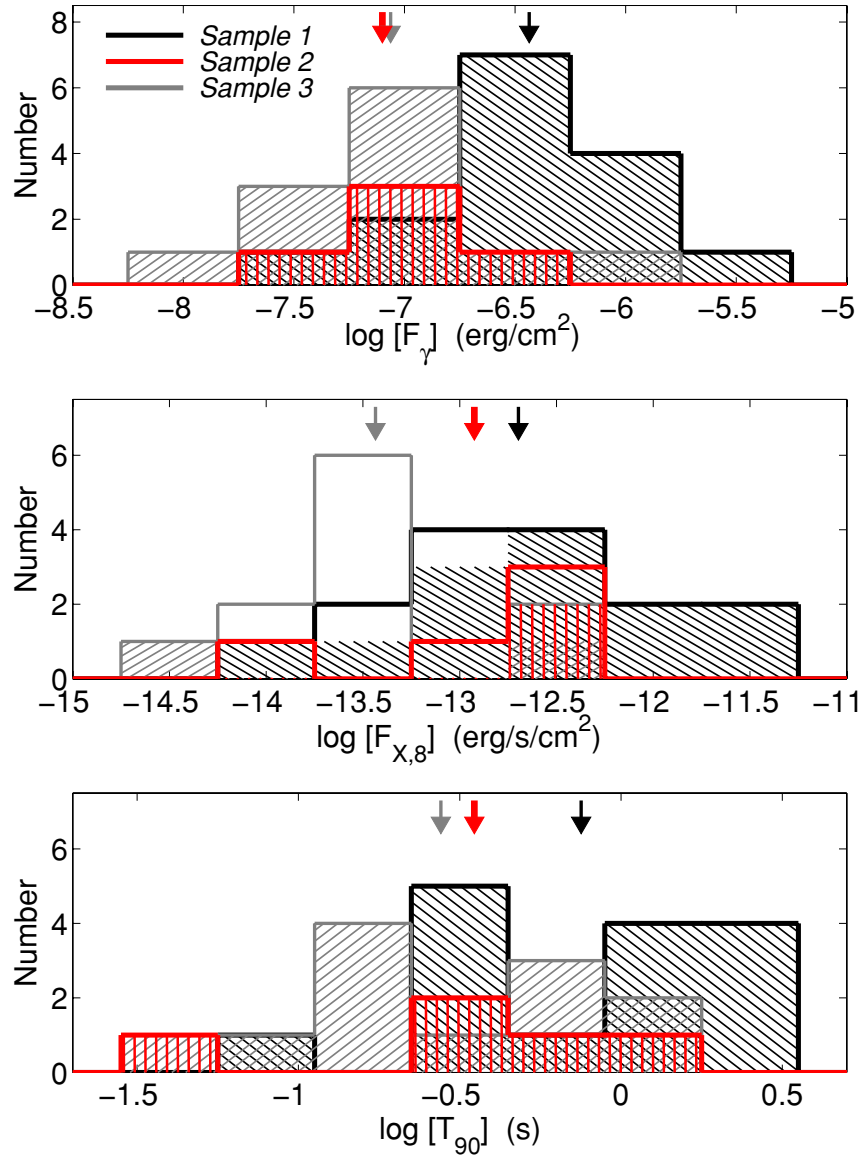

Figure 11. Histograms of $\gamma$-ray fluence (top), afterglow X-ray flux at $8 \mathrm{hr}$ (middle), and duration (bottom) for the three short GRB samples discussed in this paper. Unhatched regions mark upper limits. The arrows mark the mean for each sample, indicating that the bursts in Sample 2 and Sample 3 have lower $\gamma$-ray fluences, fainter X-ray fluxes, and shorter durations, than the bursts with detected afterglows and coincident hosts.

(A color version of this figure is available in the online journal.)

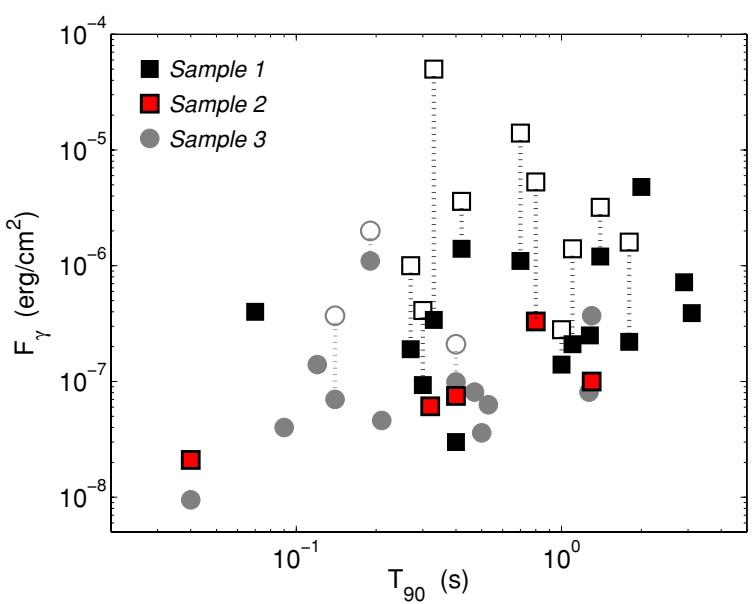

Figure 12. Short GRB $\gamma$-ray fluence as a function of duration for the three samples discussed in this paper. The filled symbols mark the $\gamma$-ray fluences measured by Swift in the $15-150 \mathrm{keV}$ energy range, while open symbols connected by dotted lines indicate fluences measured by other satellites with a broader energy range (e.g., Konus-Wind, Fermi). An overall correlation is apparent in the data. The bursts in Sample 2 and Sample 3 appear to lie below the mean correlation for the bursts in Sample 1, i.e., they have lower fluences for their durations, or longer durations for their fluences.

(A color version of this figure is available in the online journal.)

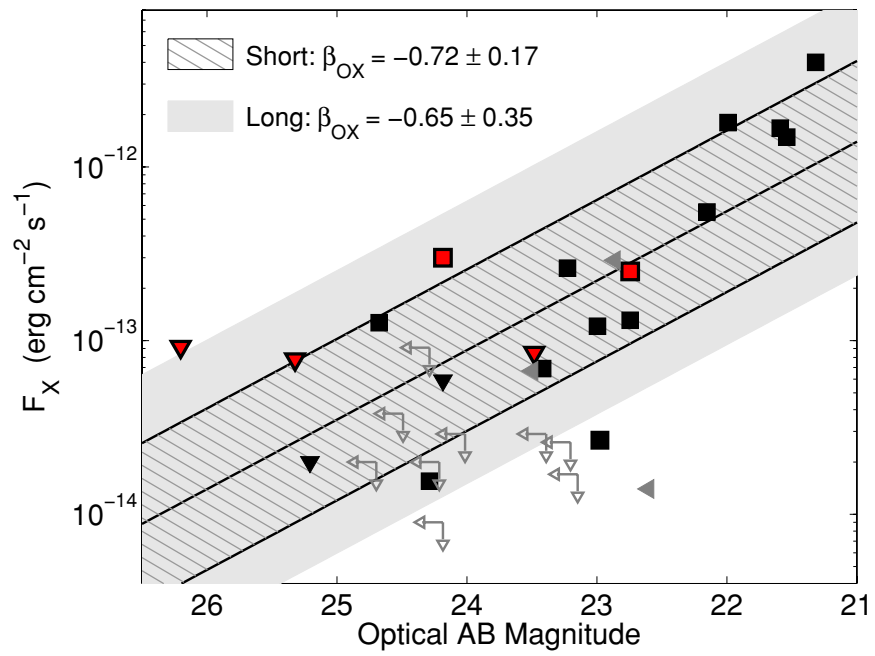

Figure 13. X-ray vs. optical flux for the bursts from Figure 9. The cross-hatched region marks the median and standard deviation assuming the expected powerlaw correlation with an index $\beta_{\mathrm{OX}}$. The light shaded region marks the region occupied by long GRBs (Jakobsson et al. 2004). The distributions for long and short GRBs are largely indistinguishable, as are the distributions for short GRBs with and without coincident hosts. We note that a large fraction of the bursts with optical upper limits also have undetected X-ray afterglows on timescales of a few hours after the burst. The overall similarity between the ratio of optical to X-ray flux for long and short GRBs does not allow us to clearly locate the synchrotron cooling frequency $\left(v_{c}\right)$ in relation to the $\mathrm{X}$-ray band. If $v_{c}>n u_{X}$ for short GRBs, the faintness of the optical afterglows for bursts with no coincident hosts cannot be used to distinguish density and redshift effects.

(A color version of this figure is available in the online journal.)

between the optical and X-ray bands and a model with $v_{c}>v_{\mathrm{X}}$ is $\Delta \beta_{\mathrm{OX}} \approx 0.25$. On the other hand, the scatter resulting from a range of $p=2.2-2.5$ is of the same order, $\Delta \beta_{\mathrm{OX}}=0.15$. Similarly, the nearly equivalent median $\beta_{\mathrm{OX}}$ values may indicate that for both GRB populations $v_{c}>v_{\mathrm{X}}$. In this case, the resulting lower limits on $v_{c}$ therefore prevent the use of $\beta_{\mathrm{OX}}$ as an indicator of density.

Comparing Sample 1 and Sample 2, we find no clear difference in $\beta_{\text {OX }}$ (Figure 13). The same is true for the bursts in Sample 3, which are all consistent with the same relation given the optical upper limits and a mix of X-ray detections and upper limits. Thus, the ratio of optical to $\mathrm{X}$-ray flux does not allow us to distinguish redshift/energy and density effects between the three samples.

To summarize, the optical afterglows of short GRB without coincident hosts (or with only optical limits) are systematically fainter than those of short GRBs with coincident hosts. The same is true for their X-ray fluxes and $\gamma$-ray fluences. The fainter afterglows may reflect lower densities (by an order of magnitude), but this does not naturally explain the lower $\gamma$-ray fluences. Alternatively, the fainter afterglows and $\gamma$-ray fluences can be explained as a result of higher redshifts $(\Delta z \approx 0.5-1)$ or lower energies (by about a factor of 3).

\section{LARGE-SCALE ENVIRONMENTS}

We next turn to an analysis of the large-scale environments of the bursts in Sample 2, partly in comparison to the hosts of bursts in Sample 1. As indicated in Section 2, we place limits of $r_{\mathrm{AB}} \approx 25.4-28.5 \mathrm{mag}$ on the brightness of any galaxy underlying the five short GRB positions (Table 2; see Perley et al. 2009 for GRB 080503). These limits are several magnitudes fainter than the measured brightnesses of short GRB hosts at $z \lesssim 1$ (Sample 1). 


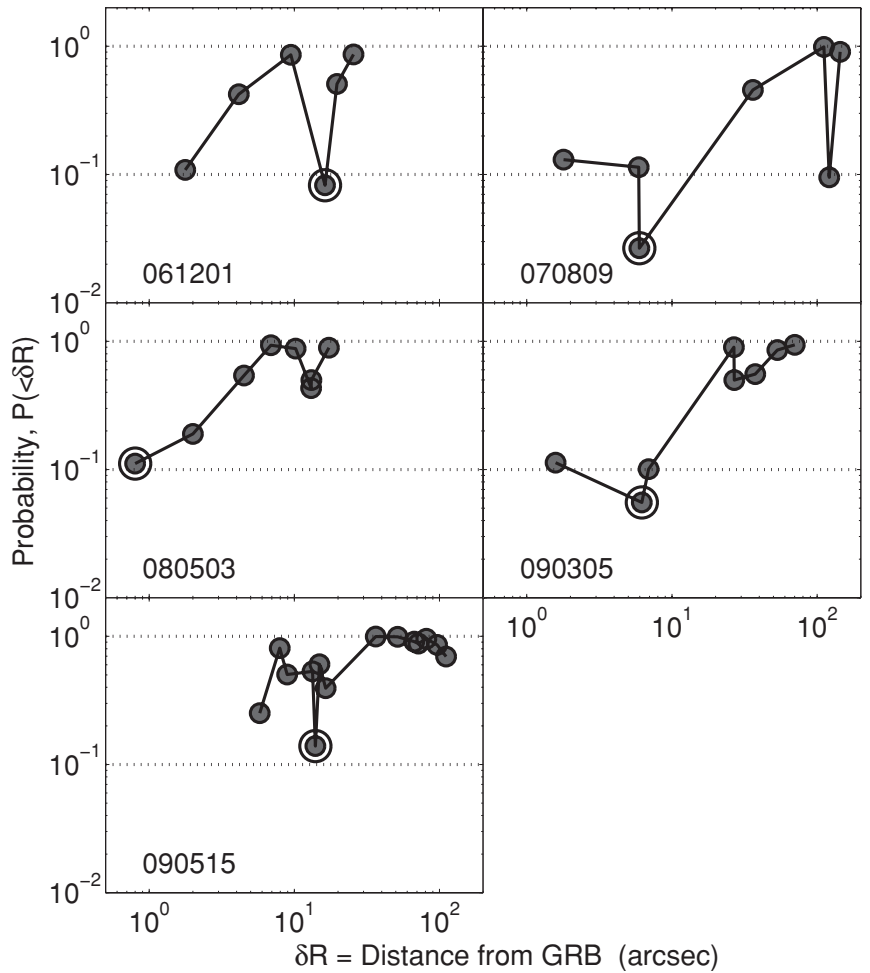

Figure 14. Probability of chance coincidence as a function of distance from a short GRB optical afterglow position for galaxies near the location of each burst. These are the galaxies marked in Figures 1-5. In each panel, we mark the galaxy with the lowest probability of chance detection with a circle. In four of the five cases, the lowest probability is associated with galaxies that are offset by $\sim 5^{\prime \prime}-15^{\prime \prime}$. Moreover, even the nearest galaxies are offset by $\approx 11^{\prime \prime} 6-5{ }^{\prime \prime} 8$.

\subsection{Probabilities of Chance Coincidence}

To assess the potential that galaxies near each of the five bursts are the hosts, we calculate their probability of chance coincidence. We follow the methodology of Bloom et al. (2002), namely, we determine the expected number density of galaxies brighter than a measured magnitude, $m$, using the results of deep optical galaxy surveys (Hogg et al. 1997; Beckwith et al. 2006):

$$
\sigma(\leqslant m)=\frac{1}{0.33 \times \ln (10)} \times 10^{0.33(m-24)-2.44} \operatorname{arcsec}^{-2}
$$
by

The probability for a given separation, $P(<\delta R)$, is then given

$$
P(<\delta R)=1-e^{-\pi(\delta R)^{2} \sigma(\leqslant m)},
$$

where we use the fact that for offsets substantially larger than the galaxy size, $\delta R$ is the appropriate radius in Equation (2) (Bloom et al. 2002).

The resulting distributions for each field are shown in Figure 14. We include all galaxies that have probabilities of $\lesssim 0.95$. We find that for four of the five bursts, faint galaxies ( 25-26 mag) can be identified within $\approx 1^{\prime \prime} .6-2^{\prime \prime}$ of the afterglow positions, with associated chance coincidence probabilities of $\approx 0.1-0.2$; in the case of GRB 090515, we do not detect any such faint galaxies within $\approx 5^{\prime \prime}$ of the afterglow position. For GRB 080503, we also include the galaxy at an offset of $0{ }^{\prime} .8$ and $m_{\mathrm{AB}}(\mathrm{F} 606 \mathrm{~W})=27.3 \pm 0.2 \mathrm{mag}$ identified by Perley et al. (2009) based on their deeper stack of HST observations. On the other hand, for four of the five bursts we find that the galaxies with the lowest probability of chance coincidence, $\approx 0.03-0.15$, are brighter objects with offsets of about $6^{\prime \prime}-16^{\prime \prime}$ from the burst

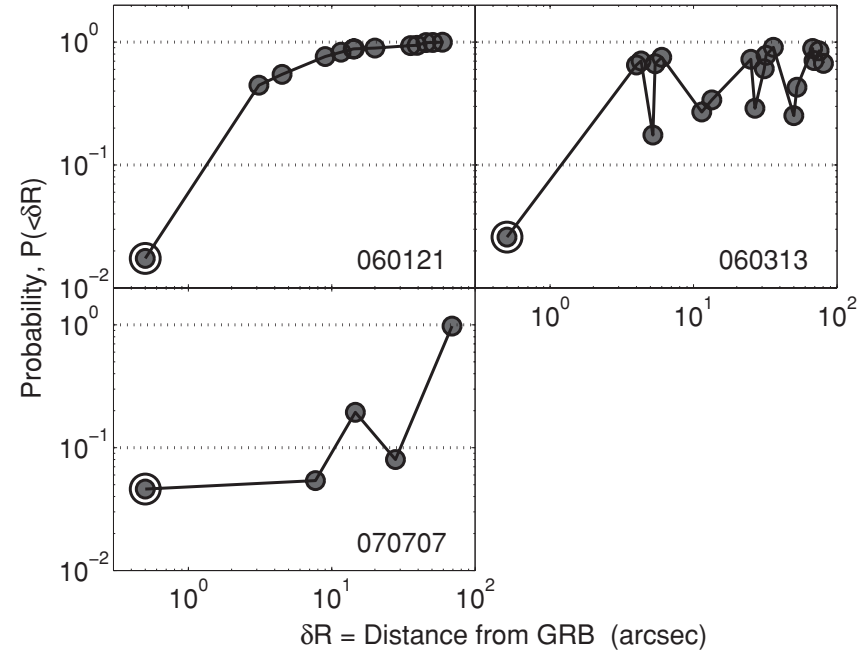

Figure 15. Same as Figure 14, but for short GRBs with coincident faint hosts In this case, the lowest probability of chance coincidence is associated with the underlying faint host.

positions; only in the case of GRB 080503 is the lowest chance coincidence associated with the nearest galaxy (see Perley et al. 2009).

For comparison, we repeat the same analysis for short GRBs from Sample 1 which have faint coincident hosts (GRB 060121: 26.0 AB mag; GRB 060313: 26.6 AB mag; and GRB 070707: $27.3 \mathrm{AB}$ mag). The results of the probability analysis are shown in Figure 15. We find that in all three cases, the coincident hosts exhibit the lowest probability of chance coincidence, $\approx 0.02-0.05$. Only in the case of GRB 070707 do we find galaxies with $\delta R \gtrsim$ few arcsec that have $P(<\delta R) \lesssim 0.1$. Thus, these three bursts are consistent with negligible offsets from faint galaxies, presumably at $z \gtrsim 1$.

The use of a posteriori probabilities to assign unique galaxy associations is fraught with difficulties. First, for a given apparent brightness, galaxies located further away from the GRB position, potentially due to larger kicks and/or longer merger timescales in the NS-NS merger framework, have higher probabilities of chance coincidence. Since we have no a priori model-independent knowledge of the range of possible kicks and merger timescales, we cannot rule out galaxies at very large offsets for which $P(<\delta R) \sim 1$. Indeed, a reasonable constraint of $v_{\text {kick }} \lesssim 10^{3} \mathrm{~km} \mathrm{~s}^{-1}$ and $\tau_{\text {merger }} \lesssim 10$ Gyr leads to only a weak constraint on the offset of $\lesssim 10 \mathrm{Mpc}$. At $z=0.1(z=1)$, this corresponds to about $1.5(0.3)$, a projected distance at which nearly all galaxies will have a chance coincidence probability of order unity.

A second difficulty is that we are using angular offsets, which ignore the potential wide range of redshifts (and by extension also luminosities) of the various galaxies. For example, if the faint galaxies with small offsets are located at $z \gtrsim 1$, the corresponding physical offsets are $\sim 15 \mathrm{kpc}$, while if the galaxies at $\sim 10^{\prime \prime}$ offsets are located at $z \sim 0.3$ (see Section 2 ), the offsets are only somewhat larger, $\sim 30 \mathrm{kpc}$. A galaxy at an even lower redshift, $z \sim 0.1$, with an offset of $50 \mathrm{kpc}$ will be located about $30^{\prime \prime}$ from the GRB position and incur a large penalty in terms of chance coincidence probability. It is important to note, however, that galaxies at lower redshift will generally have brighter apparent magnitudes, partially compensating for the larger angular separations (Equations (1) and (2)). In only a single case (GRB 070809), we find a galaxy with $P(<\delta R) \lesssim 0.1$ at 
$\delta R \gtrsim 1^{\prime}$ (which at $z=0.043$ for this galaxy corresponds to a physical offset of about $100 \mathrm{kpc}$ ).

A final complication, which is not unique to this subset of events, is that we can only measure projected offsets, $\delta R=\delta R_{3 \mathrm{D}} \times \cos (\theta)$. The measured offsets can be used as lower limits on the actual offsets, while for the overall distribution we can apply an average correction factor of $\pi / 2$, based on the expectation value for the projection factor, $\cos (\theta)$.

Despite these caveats, we can address the probability that all of the associations are spurious. This joint probability is simply the product of the individual probabilities (Bloom et al. 2002). For the faint galaxies at small angular separations the probability that all are spurious associations is $P_{\text {all }} \approx 8 \times 10^{-5}$, while for the galaxies with the lowest probability of chance coincidence the joint probability is nearly 30 times lower, $P_{\text {all }} \approx 3 \times 10^{-6}$. Conversely, the probabilities that none of the associations are spurious are $\approx 0.42$ and $\approx 0.59$, respectively. These values indicate that some spurious coincidences may be present for Sample 2. Indeed, the probabilities that 1, 2 , or 3 associations are spurious are $[0.40,0.15,0.027]$ and $[0.34,0.068,0.006]$, respectively. These results indicate that for the faint galaxies it is not unlikely that $2-3$ associations (out of 5) are spurious, while for the brighter galaxies 1-2 associations may be spurious. This analysis clearly demonstrates why a joint statistical study is superior to case-by-case attempts to associate short GRBs with galaxies at substantial offsets.

We therefore conclude that despite the weaknesses inherent to a posteriori probabilities, we conclude that there is stronger statistical support for an association of at least some of the five bursts in Sample 2 with bright galaxies at separations of $\sim 10^{\prime \prime}$, than for an association with the faint galaxies at separations of $\sim 2^{\prime \prime}$. Clearly, we cannot rule out the possibility that in reality the hosts are a mix of faint and bright galaxies with a range of angular offsets of $\gtrsim 2^{\prime \prime}$. We note that if deeper observations eventually lead to the detection of underlying galaxies $\left(\lesssim 00^{\prime} .5\right)$ at the level of $\approx 27 \mathrm{mag}$, the associated chance coincidence probabilities will be $\approx 0.05$ per object, and the joint probabilities will be only slightly higher than for the bright galaxies with $\sim 10^{\prime \prime}$ offsets. On the other hand, if we can achieve magnitude limits of $\gtrsim 28 \mathrm{mag}$ on any coincident hosts, the resulting probabilities of chance coincidence will be larger than for the offset bright galaxies. Thus, eliminating the possibility of underlying hosts at the level of $\gtrsim 28 \mathrm{mag}$ is of the utmost importance. So far, only GRB 080503 has been observed to such a depth with no detected coincident host (Perley et al. 2009), but observations of the full sample are required for a robust statistical comparison. As we discuss in Section 4.2, such deep limits will also reduce the probability of underlying hosts based on redshift arguments.

Beyond the use of projected angular offsets, we note that the faint galaxies near the burst positions are likely to have projected physical offsets of about $15 \mathrm{kpc} .^{5}$ To assess the projected physical offsets for the galaxies with the lowest probability of chance coincidence, we measured spectroscopic redshifts in three cases (GRBs 061201, 070809, and 090515; Section 2). In the case of GRB 061201, the galaxy redshift of $z=0.111$ leads to a projected physical offset of $32.4 \mathrm{kpc}$ (Fong et al. 2010). In the case of GRB 070809 , the lowest probability of chance

\footnotetext{
5 Although the redshifts of these galaxies are not known, the angular diameter distance is nearly independent of redshift beyond $z \sim 1$, which is appropriate for these faint host galaxies.
}

coincidence is not associated with the star-forming galaxy ${ }^{6}$ at $z=0.218$ identified by Perley et al. (2008), but instead belongs to the early-type galaxy identified here, which has a redshift of $z=0.473$, and hence an offset of $34.8 \mathrm{kpc}$. Finally, for GRB 090515, the lowest probability of chance coincidence is associated with a cluster early-type galaxy at $z=0.403$, leading to a physical offset of $75 \mathrm{kpc}$.

Thus, two scenarios emerge from our investigation of the large-scale environments: (1) the bursts are spatially coincident with currently undetected galaxies (with $r_{\mathrm{AB}} \gtrsim 26 \mathrm{mag}$ ), or (2) the bursts have substantial offsets of at least $\approx 15-75 \mathrm{kpc}$ depending on whether they are associated with faint galaxies at small angular separations, or brighter galaxies at $z \sim$ $0.1-0.5$; for the ensemble of five events the larger offsets are statistically more likely than the $\sim 15 \mathrm{kpc}$ offsets. In the context of large offsets, even larger values may be possible if the bursts originated in galaxies with larger separations and $P(<\delta R) \sim 1$.

These two scenarios echo the possibilities that emerged from the analysis of the afterglow and prompt emission properties (Section 3). Distinguishing between these possibilities is clearly of fundamental importance to our understanding of short GRBs: the former scenario will point to a population of very faint hosts (likely at high redshifts), while the latter scenario will provide evidence for large offsets (due to kicks or a globular cluster origin) and hence NS-NS/NS-BH progenitors for at least some short GRBs.

\subsection{Scenario 1: Undetected Faint Hosts at High Redshift}

We can place upper limits on the redshifts of the GRBs in Sample 2 based on their detections in the optical (i.e., the lack of complete suppression by the Ly $\alpha$ forest). The afterglow of GRB 061201 was detected in the ultraviolet by the Swift/UVOT and it is therefore located at $z \lesssim 1.7$ (Roming et al. 2006). The remaining four bursts were detected in the optical $g$ or $r$ band, and can therefore be placed at $z \lesssim 3$ or $\lesssim 4.3$ (Table 1 ).

We place additional constraints on the redshifts of any underlying hosts using the existing sample short GRB host galaxies. In Figure 16, we plot the $r$-band magnitudes as a function of redshift for the all available short GRB hosts from Sample 1. For the faint hosts without known redshifts (GRBs 060121, 060313, and 070707), we place upper limits on the redshift using optical detections of the afterglows (Table 1). A wide range of host magnitudes, $r_{\mathrm{AB}} \sim 16.5-27.5 \mathrm{mag}$, is apparent. We also plot the $r$-band magnitudes of long GRB hosts (Savaglio et al. 2009), as well as the $r-z$ phase space that is traced by galaxies with luminosities of $L=0.1-1 L^{*}$. We use the appropriate value of $L^{*}$ as a function of redshift, taking into account the evolving galaxy luminosity function (Steidel et al. 1999; Blanton et al. 2003; Willmer et al. 2006; Reddy \& Steidel 2009). We find excellent correspondence between the hosts of long and short GRBs, and the phase space traced by $0.1-1 L^{*}$ galaxies, at least to $z \sim 4$. In the context of these distributions, the available limits for the short GRBs in Sample 2 translate to redshifts of $z \gtrsim 1.5$ if they are $0.1 L^{*}$ galaxies, or $z \gtrsim 3$ if they are $L^{*}$ galaxies. The latter lower limits are comparable to the redshift upper limits inferred from the afterglow detections. We note that for GRB 080503 , the limits of $\gtrsim 28.5 \mathrm{mag}$ and $z \lesssim 3$ from the afterglow (Perley et al. 2009) place even more stringent limits on the luminosity of an underlying galaxy of $\lesssim 0.1 L^{*}$ galaxy.

\footnotetext{
6 We note that even if the burst was associated with this galaxy, the corresponding offset would be $20.6 \mathrm{kpc}$.
} 


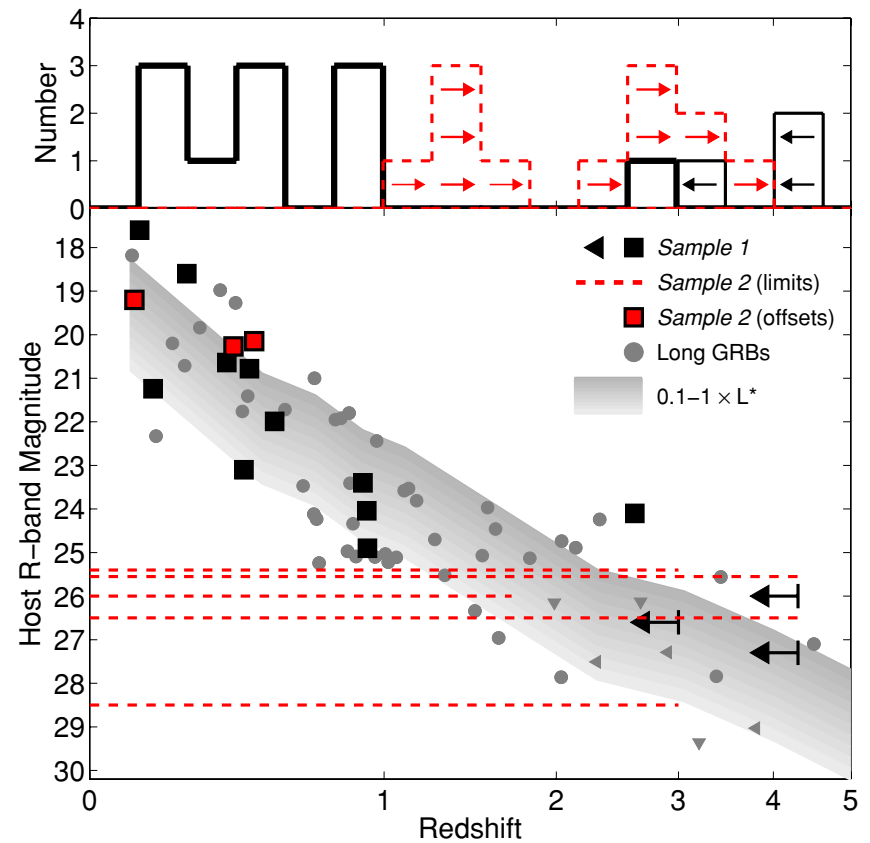

Figure 16. Main panel: host galaxy optical magnitude as a function of redshift for short GRB hosts (black squares), long GRB hosts (detections: gray circles; non-detections or no redshifts: gray triangles), and galaxies with a luminosity of $0.1-1 L^{*}$ (shaded region). The dashed lines mark the upper limits at the GRB positions for the short GRBs with no coincident hosts. The arrows mark the upper limits on the redshifts of three bursts in Sample 1 with faint hosts, based on the detection of the afterglows in the optical band (i.e., lack of a strong Lyman break). If underlying host galaxies exist for Sample 2, their non-detection indicates $z \gtrsim 1.5$ (for $0.1 L^{*}$ ) or $\gtrsim 3$ (for $L^{*}$ ). The alternative possibility that they are located at similar redshifts to the detected hosts, requires $\lesssim 0.01 L^{*}$, but this does not naturally explain their fainter afterglows. Upper panel: projected redshift histogram for Sample 1 (black) and Sample 2 (dashed red limits) under the assumption that the hosts are $0.1 L^{*}$ galaxies $(z \sim 1.5)$ and $L^{*}$ galaxies $(z \sim 3)$.

(A color version of this figure is available in the online journal.)

The redshifts of $z \gtrsim 1.5$ for putative $0.1 L^{*}$ hosts are consistent with the faintness of the optical afterglows, from which we inferred $\Delta z \approx 0.5-1$ compared to Sample 1 (Section 3). We note, however, that the one known short GRB at $z \gtrsim 2$ (GRB 090426; Antonelli et al. 2009; Levesque et al. 2010) has a host galaxy luminosity of $\sim 2 L^{*}$, which may suggest that the appropriate redshift lower limits are $z \gtrsim 3$.

The possibility that the five bursts originated at $z \gtrsim 3$ leads to a bimodal redshift distribution (Figure 16). Nearly all of the bursts in Sample 1 with a known redshift $(9 / 10)$ have $z \approx 0.2-1$, with a median of $\langle z\rangle \approx 0.5$; the sole exception is GRB 090426 at $z=2.61$. The three bursts with faint coincident hosts have upper limits of $z \lesssim 4$ from afterglow detections, while lower limits of $z \gtrsim 1.5-2$ can be placed on these hosts if they have $L \gtrsim 0.1 L^{*}$. Adding the Sample 2 bursts with the assumption that they have $z \gtrsim 3$ will furthermore result in a population of short GRBs with a median of $z \sim 3$, and leave a substantial gap at $z \sim 1-2$ (Figure 16). If the five bursts are instead hosted by $0.1 L^{*}$ galaxies, the inferred lower limits on the redshifts $(z \gtrsim 1.5)$ lead to a potentially more uniform redshift distribution.

It is difficult to explain a bimodal redshift distribution with a single progenitor population such as NS-NS binaries, without appealing to, for example, a bimodal distribution of merger timescales. Another possibility is two distinct progenitor populations, producing bursts of similar observed properties but with distinct redshift ranges. While these possibilities are

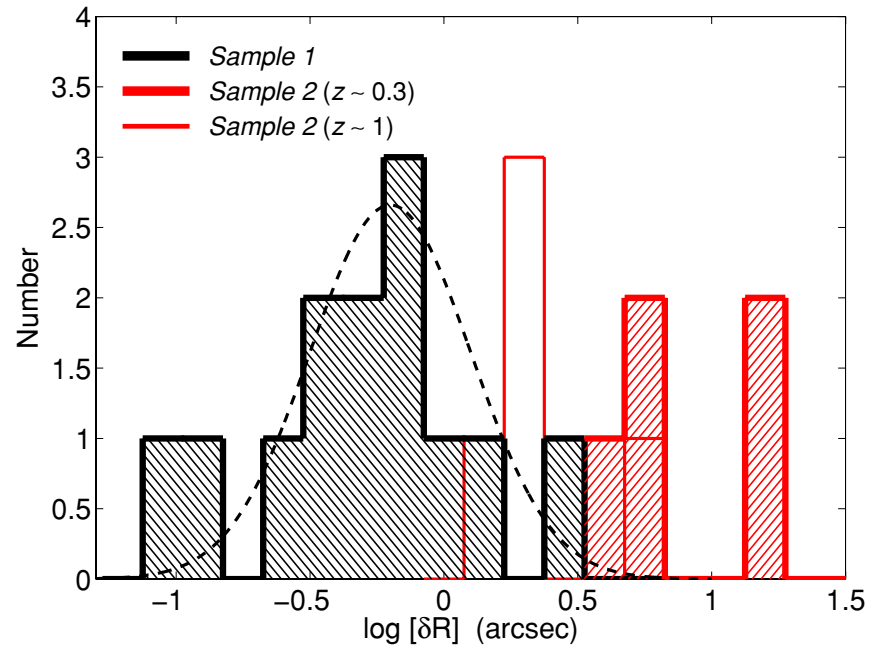

Figure 17. Histogram of projected angular offsets relative to the host galaxy center for short GRBs with coincident hosts (hatched black), and bursts with no coincident hosts if the galaxies with lowest chance coincidence probability are the hosts (hatched red), or if the faint galaxies with smallest angular separation are hosts (open red), see Figure 14. The dashed line is a log-normal fit to the bursts with coincident hosts.

(A color version of this figure is available in the online journal.)

difficult to exclude, they do not provide a natural explanation for the short GRB population.

A final alternative explanation is that any underlying hosts reside at similar redshifts to the known hosts in Sample 1 $(z \sim 0.5)$, but have significantly lower luminosities of $\lesssim 0.01$ $L^{*}$. This scenario would not naturally explain why the bursts in Sample 2 have fainter optical and X-ray afterglows, as well as lower $\gamma$-ray fluences. We therefore do not consider this possibility to be the likely explanation.

\subsection{Scenario 2: Large Offsets}

While higher redshifts may explain the lack of detected hosts, the fainter afterglows, and the weaker $\gamma$-ray fluences of the bursts in Sample 2, this scenario suffers from several difficulties outlined above. The alternative explanation is that the bursts occurred at significant offsets relative to their hosts, and hence in lower density environments that would explain the faint afterglow emission (though possibly not the lower $\gamma$-ray fluences). As we demonstrated in Section 4.1, the offsets may be $\sim 2^{\prime \prime}(\sim 15 \mathrm{kpc})$ if the bursts originated in the faint galaxies at the smallest angular separations, or $\sim 10^{\prime \prime}(\sim 30-75 \mathrm{kpc})$ if they originated in the brighter galaxies with the lowest probability of chance coincidence. Below we address the implications of these two possible offset groups through a comparison to the offsets measured for the bursts in Sample 1 (e.g., Fong et al. 2010).

We plot the distributions of projected angular offsets for the short GRBs with and without coincident hosts in Figure 17. The offsets for Sample 1 have a mean and standard deviation of about $0.7 \pm 0.7$ and a range of about $00^{\prime \prime} 1-3^{\prime \prime}$. Modeled with a log-normal distribution, the resulting mean and width in units of arcseconds are $\log (\delta R) \approx-0.2$ and $\sigma_{\log (\delta R)} \approx 0.35$. If we associate the short bursts in Sample 2 with the galaxies that have the lowest probability of chance coincidence, the resulting distribution has $\langle\delta R\rangle=9 \pm 6$ arcsec. This is clearly distinct from the distribution for Sample 1. The effect is less pronounced if we associate the bursts with the galaxies located at the smallest angular offsets (Figure 17). Even for these separations the mean and standard deviation $\operatorname{are} \log (\delta R) \approx 2.6 \pm 1.8$ arcsec. 


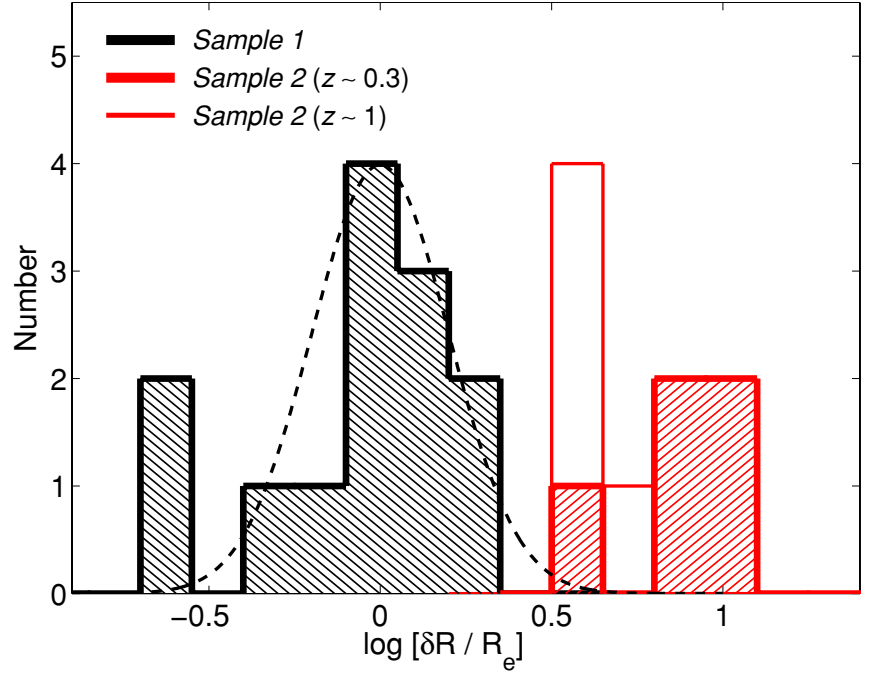

Figure 18. Same as Figure 17 but normalized relative to the host effective radii, $R_{e}$. The dashed line is a log-normal fit to the bursts with coincident hosts. (A color version of this figure is available in the online journal.)

As noted in Section 4.1, the projected angular distances may not be the most robust quantity for measuring the offset distribution. An alternative quantity is the offset normalized by each host's effective radius (Fong et al. 2010). This quantity takes into account the varying sizes of the hosts due to both intrinsic size variations and redshift effects. It also gives a better indication of whether the burst coincides with the host light or is significantly offset. As shown in Figure 18, the host-normalized offsets of Sample 1 have a mean and standard deviation of about $1 \pm 0.6 R_{e}$, and a range of about $0.2-2 R_{e}$. A $\log$-normal fit results in a mean of $\log \left(\delta R / R_{e}\right) \approx 0$ and a width of $\sigma_{\log \left(\delta R / R_{e}\right)} \approx 0.2$. The bursts in Sample 2 have much larger host-normalized offsets, with $\left(\delta R / R_{e}\right)=7.3 \pm 2.3$ if they originated in the galaxies with the lowest chance coincidence probability. Even if we associate the bursts with the nearest faint hosts, the distribution has a mean of about $4 R_{e}$, reflecting the fact that the effective radii of the faint galaxies are smaller than those of the brighter ones.

Finally, we plot the projected physical offsets in Figure 19. The mean and standard deviation for Sample 1 are $\delta R=$ $4.2 \pm 3.8 \mathrm{kpc}$, and a log-normal fit results in a mean of $\log (\delta R) \approx 0.5$ and a width of $\sigma_{\log (\delta R)} \approx 0.3$. On the other hand, the bursts in Sample 2 have a mean offset of about $19 \mathrm{kpc}$ if they arise in the faint galaxies with small angular separation, or about $40 \mathrm{kpc}$ if they arise in the brighter galaxies, again pointing to distinct distributions.

The distributions of angular, physical, and host-normalized offsets exhibit a clear bimodality if we associate the bursts in Sample 2 with the galaxies at $z \sim 0.1-0.5$. This is particularly apparent in the more meaningful quantities, namely, physical and host-normalized offsets (Figures 18 and 19). The effect is still apparent, though less pronounced, in the case of association with the faint galaxies at $z \gtrsim 1$. Thus, if the offset scenario is correct, the resulting distributions point to a possible bimodality rather than a single continuous distribution of offsets.

The cumulative distributions of physical offsets for Sample 1 alone, and in conjunction with the two possible offset groups for Sample 2, are shown in Figure 20. The combined distributions have a median of about $4 \mathrm{kpc}$, driven by the bursts with coincident hosts. However, there is a clear extension to larger physical offsets in the case of association with the brighter

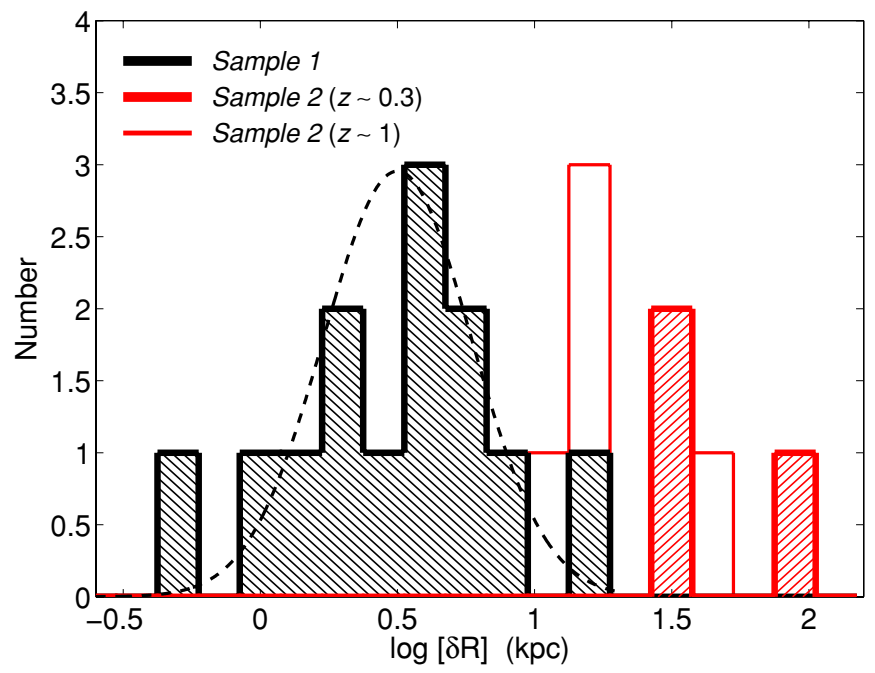

Figure 19. Same as Figure 17 but projected physical offsets in units of kpc. The dashed line is a log-normal fit to the bursts with coincident hosts.

(A color version of this figure is available in the online journal.)

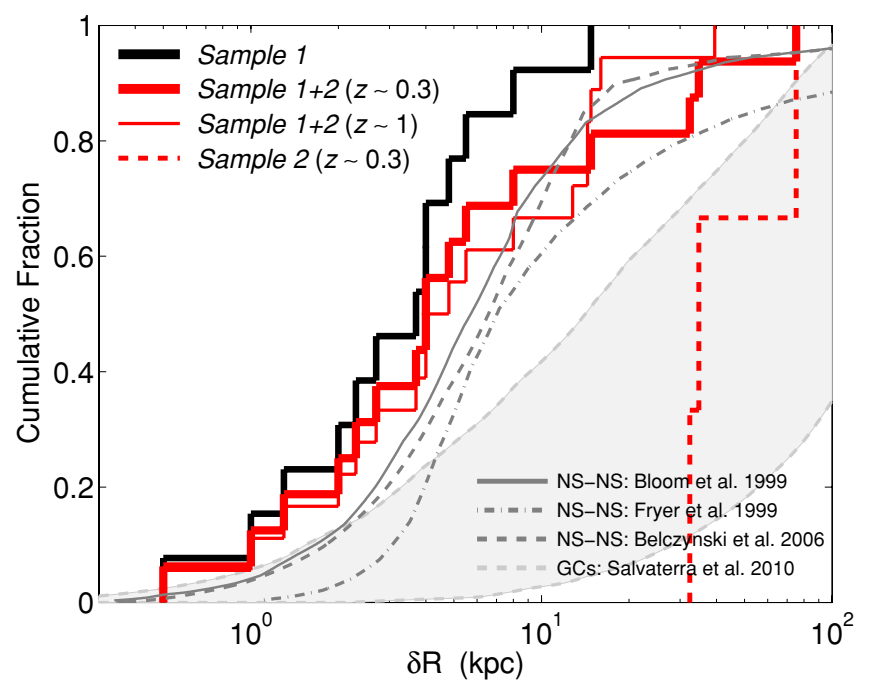

Figure 20. Cumulative distributions of projected physical offsets for short GRBs with coincident hosts (black line), and combined with offsets for the hosts with the lowest probabilities of chance coincidence (thick red line) or the faint hosts with smallest angular offsets (thin red line). Also shown are predicted distributions for NS-NS kicks from several models (Bloom et al. 1999; Fryer et al. 1999; Belczynski et al. 2006), and for dynamically formed NS-NS binaries from globular clusters (shaded region marks a range of predictions for host galaxy masses of $5 \times 10^{10}-10^{12} M_{\odot}$; Salvaterra et al. 2010). The models with kick velocities are in good agreement with the measured offset distribution for either set of galaxy associations, while the globular clusters model provides a poor match to the data.

(A color version of this figure is available in the online journal.)

galaxies, with about $20 \%$ of all objects having $\delta R \gtrsim 30 \mathrm{kpc}$. The cumulative distributions are particularly useful for comparison with NS-NS merger models since predictions exist for both the kick scenario and the globular cluster origin model. We turn to this discussion below.

\section{DISCUSSION AND IMPLICATIONS}

We have shown that the lack of host galaxy detections in coincidence with the bursts in Sample 2 indicates either a high-redshift $(z \gtrsim 2)$ origin, or substantial projected offsets of $\sim 15-75 \mathrm{kpc}\left(\approx 4-10 R_{e}\right)$. Both scenarios can account for the 
lack of underlying galaxy detections and the fainter optical/ $\mathrm{X}$-ray afterglows (either due to redshift or density effect); the possibility of large offsets from galaxies at $z \sim 0.1-0.5$, however, does not naturally explain the lower $\gamma$-ray fluences and somewhat shorter durations of the bursts in Sample 2. Indeed, the lower fluences can be more easily accommodated in the case of undetected high-redshift coincident hosts, or in the case of faint hosts with offsets of $\sim 15 \mathrm{kpc}$.

Below we test the results of the offset scenario against predictions for NS-NS merger models and dynamically formed NS-NS binaries in globular clusters. We also investigate whether the densities expected at offsets of $\sim 15-75 \mathrm{kpc}$ (i.e., galaxy halos or the IGM) can accommodate the observed optical magnitudes. Finally, we discuss the implications of the various scenarios for short GRB energetics.

\subsection{Comparison to NS-NS Merger Models}

The sample of short GRBs with optical afterglows represents about $1 / 3$ of all short bursts, and may thus not be fully representative. One often-discussed bias is that the bursts with optical afterglows require a high circumburst density, and therefore have negligible offsets. However, from our analysis in this paper it is clear that one explanation for the lack of coincident hosts for the bursts in Sample 2 is indeed large offsets, despite their detection in the optical band.

The bursts with only X-ray afterglow detections (Swift/ XRT) have typical positional uncertainties of $\sigma_{X} \approx 2^{\prime \prime}-5^{\prime \prime}$ and therefore lead to a deeper ambiguity about the identity of the hosts. We have previously argued that most of these bursts are associated with galaxies consistent with a negligible offset (or as large as tens of kpc; Berger et al. 2007; Fong et al. 2010). In some cases, larger offsets have been advocated based on a posteriori chance coincidence probabilities (e.g., GRB 050509b: Bloom et al. 2006; GRB 060502b: Bloom et al. 2007). However, from the analysis in Section 4.1 it is clear that while the inferred probabilities are only weakly dependent on the positional accuracy when $\delta R \gtrsim \sigma_{X}$ (i.e., galaxies at large offsets), a large penalty is incurred for faint galaxies located within the X-ray error circles since in that case the appropriate value in Equation (2) is $\delta R=\sigma_{X}$. As a result, the faint galaxies will have chance coincidence probabilities of $P(<\delta R) \sim 1$. To avoid this complication in our comparison to NS-NS model predictions, we restrict the analysis to the sample with optical afterglow positions.

In a recent work by Fong et al. (2010), we have shown that the offset distribution for short GRBs with optical and/ or X-ray positions, and accounting for incompleteness due to bursts with only $\gamma$-ray positions, is broadly consistent with the predictions of NS-NS merger models (Bloom et al. 1999; Fryer et al. 1999; Belczynski et al. 2006). In particular, we concluded that $\gtrsim 25 \%$ of all short bursts have $\delta R \lesssim 10 \mathrm{kpc}$, while $\gtrsim 5 \%$ have offsets of $\gtrsim 20 \mathrm{kpc}$. We repeat the analysis here using the offsets inferred in Section 4.3 for both the faint galaxies at small angular separations and the brighter galaxies at larger separations. As shown in Figure 20, the model predictions have a median of about $6 \mathrm{kpc}$, compared to about $4 \mathrm{kpc}$ for the observed sample. On the other hand, the models predict $10 \%-20 \%$ of offsets to be $\gtrsim 30 \mathrm{kpc}$, in good agreement with the observed distribution in both the $\sim 15 \mathrm{kpc}$ and $\sim 40 \mathrm{kpc}$ scenarios. We note that the overall smaller offsets measured from the data may be due to projection effects. Indeed, the mean correction factor of $\pi / 2$ nicely reconciles the theoretical and observed distributions.
In the previous section we noted a bimodality in the physical and host-normalized offsets for Sample 1 and Sample 2 (Figures 18 and 19). In the framework of NS-NS binary kicks, this bimodality may indicate that the binaries generally remain bound to their host galaxies, thereby spending most of their time at the maximal distance defined by $d_{\max }=2 G M_{\text {host }} / v_{\text {kick }}^{2}$ (i.e., with their kinetic energy stored as potential energy; Bloom et al. 2007). This would require typical kick velocities of less than a few hundred $\mathrm{km} \mathrm{s}^{-1}$.

We further compare the observed offset distribution to predictions for dynamically formed NS-NS binaries in globular clusters, with a range of host galaxy virial masses of $5 \times 10^{10}-10^{12} M_{\odot}($ Salvaterra et al. 2010). These models predict a range of only $\approx 5 \%-40 \%$ of all NS-NS mergers to occur within $10 \mathrm{kpc}$ of the host center, in contrast to the observed distribution with about $70 \%$ with $\delta R \lesssim 10 \mathrm{kpc}$. We stress that this result is independent of what offsets we assign to the bursts in Sample 2 since they account for only $1 / 4$ of the bursts with optical afterglows. On the other hand, the globular cluster origin may account for the bimodality in the physical and host-normalized offsets (Figures 18 and 19), with the objects in Sample 2 arising in globular clusters and the objects with coincident hosts arising from primordial NS-NS binaries. This possibility also agrees with the predicted fraction of dynamically formed NS-NS binaries of $\sim 10 \%-30 \%$ (Grindlay et al. 2006). The cumulative offset distributions for Sample 2 alone (assuming the hosts are the galaxies with the lowest probability of chance coincidence) is well matched by the range of predictions for dynamically formed NS-NS binaries in globular clusters (Figure 20). In this scenario, however, the implication is that short GRBs outside of globular clusters do not experience kicks as expected for NS-NS binaries since the largest measured offset is only $15 \mathrm{kpc}$.

Unless the populations of short GRBs with only X-ray or $\gamma$-ray positions have fundamentally different offset distributions, we conclude that the measured offsets of short GRBs and the predicted offsets for NS-NS kicks are in good agreement, if we treat all short GRBs with optical afterglows as a single population. Alternatively, it is possible that the bimodal distributions of physical and host-normalized offsets point to a progenitor bimodality, with the bursts in Sample 2 originating in globular clusters.

\subsection{Circumburst Densities}

Large offsets are expected to result in low circumburst densities, and we now investigate whether the observed optical magnitudes for Sample 2 can be used to place meaningful constraints on the offsets. The optical afterglow magnitudes do not provide a direct measure of the circumburst density since they depend on a complex combination of density, energy, and fractions of the burst energy imparted to the radiating electrons $\left(\epsilon_{e}\right)$ and magnetic fields $\left(\epsilon_{B}\right)$. From our comparison of the X-ray and optical afterglows (Section 3), we were unable to clearly locate the cooling frequency, which in principle can provide additional constraints on the density. However, we can still gain some insight into the required circumburst densities using a few basic assumptions. We assume that the $\gamma$-ray energy $\left(E_{\gamma, \text { iso }}\right)$ is a reasonable proxy for the total energy, that $\epsilon_{e}, \epsilon_{B}<1 / 3$, and that $p=2.2$. We further use a fiducial redshift of $z=0.5$ (Figure 16) and a fiducial observation time of $8 \mathrm{hr}$ (Section 3 ).

To determine $E_{\gamma, \text { iso }}$, we use the $15-150 \mathrm{keV}$ fluences measured by Swift (Table 1), and determine a correction factor to account for the incomplete energy coverage using bursts that have also been detected by satellites with a broader energy 


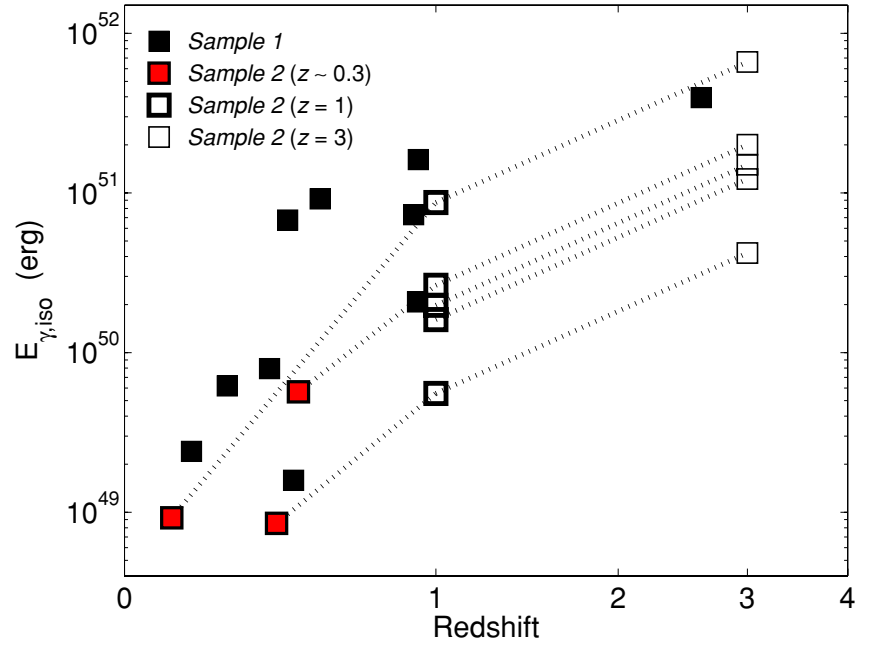

Figure 21. Isotropic-equivalent $\gamma$-ray energy as a function of redshift for the bursts with detected optical afterglows. We plot the inferred energies for Sample 2 at the redshifts corresponding to the galaxies with the lowest probability of chance coincidence (red squares), at $z \sim 1$ corresponding to the faint galaxies with the smallest angular separations (thick open squares), and at $z \sim 3$ corresponding to the case of undetected underlying hosts with a luminosity of about $L^{*}$ (thin open squares). The bursts in Sample 2 match the distribution for the Sample 1 bursts at any of these redshift intervals.

(A color version of this figure is available in the online journal.)

range (Figure 12 ). We find a typical correction factor of $\approx 5$ for the $\approx 10-10^{3} \mathrm{keV}$ range. For the fiducial redshift of $z=0.5$, the mean energy for short GRBs with optical afterglows is $E_{\gamma \text {,iso }} \approx 2 \times 10^{51} \mathrm{erg}$, when we include the correction factor (Figure 21).

With these assumptions, we find the following relation between the optical brightness and the circumburst density (Granot \& Sari 2002):

$$
F_{v, \text { opt }}<1 \mathrm{mJy} \times n^{1 / 2} \text {. }
$$

Given the typical observed fluxes of about $2 \mu \mathrm{Jy}$ for Sample 1 , and about $0.6 \mu \mathrm{Jy}$ for Sample 2, we infer typical minimum densities of $\gtrsim 4 \times 10^{-6}$ and $\gtrsim 4 \times 10^{-7} \mathrm{~cm}^{-3}$, respectively. Thus, the observed optical brightnesses can be produced even at very low densities that are typical of the IGM. This indicates that we cannot rule out the large offset scenario based on density arguments. Indeed, even if we allow for more typical values of $\epsilon_{e} \approx \epsilon_{B} \approx 0.1$, the resulting densities are $\approx 4 \times 10^{-4}$ and $4 \times 10^{-5} \mathrm{~cm}^{-3}$, respectively. These results compare favorably with predictions for NS-NS kicks, which suggest that most mergers will occur at densities of $\gtrsim 10^{-6} \mathrm{~cm}^{-3}$ (Perna \& Belczynski 2002; Belczynski et al. 2006). The distribution of densities in globular clusters is not well known, preventing a meaningful comparison to our inferred minimum densities (Salvaterra et al. 2010).

\subsection{Energetics}

Finally, we investigate the energetics of the bursts in Sample 2 in the context of a high-redshift origin $(z \gtrsim 3)$, an origin in the faint galaxies at $\approx 2^{\prime \prime}$ separations $(z \sim 1)$, and an origin in the galaxies with the lowest probabilities of chance coincidence $(z \approx 0.1-0.5)$. The resulting values of $E_{\gamma, \text { iso }}$ are shown in Figure 21. For the lowest redshift origin, the inferred values are $\approx(1-5) \times 10^{49} \mathrm{erg}$, for a $z \sim 1$ origin they are $\approx 5 \times 10^{49}-10^{51} \mathrm{erg}$, and for a $z \gtrsim 3$ origin they are $\approx 5 \times 10^{50}-5 \times 10^{51} \mathrm{erg}$.
For comparison, the mean value for the bursts in Sample 1 is $\left\langle E_{\gamma, \text { iso }}\right\rangle \approx 8 \times 10^{50} \mathrm{erg}$, with a range of $\approx 10^{49}-5 \times 10^{51} \mathrm{erg}$. However, there is a clear redshift dependence for the measured $E_{\gamma \text {,iso }}$ values (Figure 21 ), with $\approx 5 \times 10^{49}$ erg at $z \lesssim 0.5$ and $\approx 5 \times 10^{50}$ erg at $z \gtrsim 0.5$. Thus, the bursts in Sample 2 generally fit within the known range of isotropic energies regardless of their actual redshift. Indeed, at $z \sim 3$ they exhibit similar values of $E_{\gamma \text {,iso }}$ to that of GRB 090426. As a result, we cannot use the inferred energy release as a clear discriminant of the redshift range for Sample 2.

If the bursts in Sample 2 indeed originated at high redshifts, the resulting isotropic-equivalent energies suggest that there is either a large spread in the energy release of short GRBs (at least 2 orders of magnitude) or a large variations in the ejecta geometry. If the typical energy for short GRBs is about $5 \times 10^{49} \mathrm{erg}$, as indicated by the nearest events, ${ }^{7}$ then the inferred values of $E_{\gamma \text {,iso }} \approx 2 \times 10^{51}$ at $z \sim 3$ indicate opening angles of $\approx 10^{\circ}-15^{\circ}$. This is similar to the opening angle of about $7^{\circ}$ that was inferred for GRB 051221 (Burrows et al. 2006; Soderberg et al. 2006).

\section{CONCLUSIONS}

We undertook the first systematic study of short GRBs with detected optical afterglows (and hence subarcsecond position) but no coincident host galaxies to limits of $r_{\mathrm{AB}} \gtrsim 26$ mag. We find that the optical afterglows of these bursts are fainter by about 1.4 mag compared to the optical afterglows of short GRBs with coincident hosts. They are similarly fainter in X-rays, and have somewhat lower $\gamma$-ray fluences and slightly shorter durations. Both samples have similar ratios of X-ray to optical flux, which are moreover similar to the ratios measured for long GRBs. The fainter afterglows of the bursts lacking coincident hosts may be due to lower densities, lower energies, or higher redshifts. However, we note that only the scenarios with lower energies or higher redshifts naturally explain the faintness of the prompt emission in the context of the standard GRB model. This is because in the context of internal processes (shocks or magnetic dissipation) the external density should not play a role.

We further use deep optical imaging to determine the probability of chance coincidence for galaxies in the field around each burst, and to place redshift limits for underlying hosts under the assumption that they are drawn from the same distribution of the detected short GRB hosts $\left(L \approx 0.1-1 L^{*}\right)$. This analysis leads to the following possible scenarios: (1) the underlying hosts are fainter than $\sim 26 \mathrm{mag}$, indicative of redshifts of $z \gtrsim 1.5$ (if $L \sim 0.1 L^{*}$ ) or $z \gtrsim 3$ (if $L \sim L^{*}$ ); or (2) the hosts are galaxies with substantial offsets-either faint galaxies at separations of $\approx 2^{\prime \prime}(\approx 15 \mathrm{kpc}$ for $z \gtrsim 1)$ or brighter galaxies, which we find to be located at $z \sim 0.1-0.5$, with offsets of $\approx 5^{\prime \prime}-15^{\prime \prime}$ $(\approx 30-75 \mathrm{kpc})$. In the former scenario, unless the galaxies have $L \sim 0.1 L^{*}$, the resulting redshift distribution is bimodal with peaks at $z \sim 0.5$ and $\sim 3$. Such a scenario most likely requires a bimodal age distribution in the context of NS-NS mergers, or two distinct progenitor systems dominating at low and high redshifts. While this cannot be ruled out by present data, the lack of overlap at $z \sim 1-2$ is difficult to explain. We also conclude that it is unlikely that any faint underlying hosts are low-luminosity

\footnotetext{
7 We ignore the factor of $\sim 5$ correction to the energy, due to incomplete energy coverage (Section 5.2), since this would roughly apply to all bursts in roughly the same way. The higher redshift events may require somewhat smaller corrections due to redshifting of the spectral peak into the softer energy band covered by Swift.
} 
galaxies $\left(L \lesssim 0.01 L^{*}\right)$ at similar redshifts to the detected hosts, since this does not naturally explain the difference in afterglow and prompt emission properties.

In the context of the large offsets scenario, the probability of chance coincidence (both individually and for the sample) is lower for the brighter galaxies at offsets of $\approx 5^{\prime \prime}-15^{\prime \prime}$ than for the faint galaxies at offsets of $\approx 2^{\prime \prime}$. However, it is not unlikely that the true associations are a mix of both populations, since in each case there is a non-negligible probability that 1-2 of the associations are spurious. We note that optical positions are crucial for a uniform comparison of the chance coincidence probabilities for faint galaxies with small offsets and bright galaxies with larger offsets; in the case of only X-ray positions there is an inherent bias (in the sense of a posteriori) against associations with faint galaxies inside the error circle, despite the fact that they are consistent with no offset.

From spectroscopic observations for three of the five bursts, we find that the galaxies with the lowest probability of chance coincidence are a star-forming galaxy at $z=$ 0.111 (GRB 061201), an early-type galaxy at $z=0.473$ (GRB 070809), and an early-type cluster member galaxy at $z=0.403$ (GRB 090515). If these associations are indeed correct, they only slightly alter the host demographics, which are dominated by star-forming galaxies (Berger 2009). However, this does suggest that our present understanding of the relative ratios of star forming and elliptical hosts may be incomplete.

The resulting distributions of angular, physical, and hostnormalized offsets for the bursts with and without coincident hosts appear to be distinct, rather than continuous. However, the joint distribution of projected physical offsets is in good agreement with theoretical predictions for NS-NS binary mergers. On the other hand, the predicted distribution for dynamically formed NS-NS binaries in globular clusters provides a much poorer fit to the entire data set, unless they account for only the bursts with large offsets (Sample 2). In the case of a hybrid population of primordial and dynamically formed binaries, with the latter accounting for only the large offsets, the fact that all the remaining offsets (Sample 1) are $\lesssim 10 \mathrm{kpc}$ is indicative of no significant kicks. The large physical offsets also naturally explain the fainter afterglow emission as a result of lower circumburst densities. The resulting isotropic $\gamma$-ray energies match the observed distribution for short GRBs with coincident hosts, either at $z \sim 0.3$ or at $z \gtrsim 1$.

Our conclusion that large offsets of $\sim 15-70 \mathrm{kpc}$ (corresponding to $\approx 4-10$ galactic effective radii) are a likely explanation for the bursts with optical afterglows and no coincident hosts is of fundamental importance. The only progenitor model that naturally explains this result in the merger of NS-NS/NS-BH binaries, most likely due to kicks, or possibly with a minor contribution from a globular cluster population (accounting specifically for the events in Sample 2). While a conclusive demonstration of a large offset requires an absorption redshift measurement that matches an offset galaxy redshift, the distribution of optical afterglow magnitudes indicates that this will be difficult to achieve. Indeed, an absorption redshift is available for only one likely short GRB (Antonelli et al. 2009; Levesque et al. 2010).

We end with several important observations. First, rapid optical follow-up of short GRBs with $8 \mathrm{~m}$ class telescopes is essential since observations to a depth of about 25 mag within a few hours after the burst may recover nearly all optical afterglows, regardless of circumburst density (Figure 9). Second, short GRBs with only X-ray positions are unlikely to provide strong support for either negligible or large offsets due to the appreciable size of the error circles and the fact that the sample with optical afterglows exhibits wide host galaxy diversity, i.e., events with coincident bright hosts at $z \sim 0.2-1$, events with coincident faint hosts likely at $z \gtrsim 1$, and events with no coincident hosts likely due to offsets. Third, a meaningful study of short GRB offsets requires a statistical approach to mitigate the shortcomings of a posteriori chance coincidence probabilities, as well as to incorporate the relevant information from afterglow and prompt emission observations.

Finally, we note that from a wide range of observations of both the afterglows and host galaxies it appears that the case for NS-NS mergers as the progenitors of short GRBs is gaining observational support. Our main result here is that short GRBs with optical afterglows and no detected host galaxies are somewhat less likely to be explained with high redshifts or with dwarf galaxy hosts at low redshift. Instead, these bursts likely exploded $\sim 15 \mathrm{kpc}$ from galaxies at $z \sim 1$ or tens of $\mathrm{kpc}$ from galaxies at $z \sim 0.3$. With larger samples, these possibilities will allow us to improve our understanding of the short GRB redshift distribution, the host galaxy demographics, and predictions for gravitational wave detections. If high redshifts indeed turn out to be prevalent, this will have a significant effect on the possibility of multiple progenitor populations.

We acknowledge helpful discussions with Alicia Soderberg, Ryan Chornock, and Josh Grindlay. This work was partially supported by Swift AO5 grant number 5080010, and made use of data supplied by the UK Swift Science Data Centre at the University of Leicester.

\section{REFERENCES}

Antonelli, L. A., et al. 2009, A\&A, 507, L45

Barthelmy, S. D., et al. 2005, Nature, 438, 994

Beckwith, S. V. W., et al. 2006, AJ, 132, 1729

Belczynski, K., Perna, R., Bulik, T., Kalogera, V., Ivanova, N., \& Lamb, D. Q. 2006, ApJ, 648, 1110

Berger, E. 2006a, GRB Coordinates Network, 5952, 1

Berger, E. 2006b, in AIP Conf. Ser. 836, Gamma-Ray Bursts in the Swift Era, ed. S. S. Holt, N. Gehrels, \& J. A. Nousek (Melville, NY: AIP), 33

Berger, E. 2007, ApJ, 670, 1254

Berger, E. 2009, ApJ, 690, 231

Berger, E., Cenko, S. B., Fox, D. B., \& Cucchiara, A. 2009, ApJ, 704, 877

Berger, E., Chornock, R., Tanvir, N., Levan, A. J., Fox, D., Cucchiara, A., Fruchter, A., \& Graham, J. 2010, GRB Coordinates Network, 10395, 1 Berger, E., \& Kelson, D. 2009, GRB Coordinates Network, 8934, 1

Berger, E., Soderberg, A. M., Cucchiara, A., \& Fox, D. B. 2008, GRB Coordinates Network, 8736, 1

Berger, E., et al. 2005, Nature, 438, 988

Berger, E., et al. 2007, ApJ, 664, 1000

Blanton, M. R., et al. 2003, ApJ, 592, 819

Bloom, J. S., Kulkarni, S. R., \& Djorgovski, S. G. 2002, AJ, 123, 1111

Bloom, J. S., Sigurdsson, S., \& Pols, O. R. 1999, MNRAS, 305, 763

Bloom, J. S., et al. 2006, ApJ, 638, 354

Bloom, J. S., et al. 2007, ApJ, 654, 878

Burrows, D. N., et al. 2006, ApJ, 653, 468

Cenko, S. B., Cobb, B. E., Perley, D. A., \& Bloom, J. S. 2009, GRB Coordinates Network, 8933, 1

Cenko, S. B., et al. 2008, arXiv:0802.0874

D’Avanzo, P., et al. 2009, A\&A, 498, 711

de Ugarte Postigo, A., Kann, D. A., Castro-Tirado, A. J., Aguirre, A., \& Jordi, C. 2008, GRB Coordinates Network, 7644, 1

de Ugarte Postigo, A., et al. 2006, ApJ, 648, L83

Eichler, D., Livio, M., Piran, T., \& Schramm, D. N. 1989, Nature, 340, 126

Evans, P. A., et al. 2007, A\&A, 469, 379

Evans, P. A., et al. 2009, MNRAS, 397, 1177

Ferrero, P., et al. 2007, AJ, 134, 2118

Fong, W., Berger, E., \& Fox, D. B. 2010, ApJ, 708, 9

Fox, D. B., et al. 2005, Nature, 437, 845

Fryer, C. L., Woosley, S. E., \& Hartmann, D. H. 1999, ApJ, 526, 152 
Gal-Yam, A., et al. 2008, ApJ, 686, 408

Galeev, A., et al. 2009, GRB Coordinates Network, 9549, 1

Gehrels, N., et al. 2005, Nature, 437, 851

Gehrels, N., et al. 2008, ApJ, 689, 1161

Graham, J. F., et al. 2009, ApJ, 698, 1620

Granot, J., \& Sari, R. 2002, ApJ, 568, 820

Greco, G., et al. 2008, GRB Coordinates Network, 7977, 1

Grindlay, J., Portegies Zwart, S., \& McMillan, S. 2006, Nature Phys., 2, 116

Grupe, D., Burrows, D. N., Patel, S. K., Kouveliotou, C., Zhang, B., Mészáros, P., Wijers, R. A. M., \& Gehrels, N. 2006, ApJ, 653, 462

Hjorth, J., et al. 2005, Nature, 437, 859

Hogg, D. W., Pahre, M. A., McCarthy, J. K., Cohen, J. G., Blandford, R., Smail, I., \& Soifer, B. T. 1997, MNRAS, 288, 404

Hopman, C., Guetta, D., Waxman, E., \& Portegies Zwart, S. 2006, ApJ, 643, L91

Hurley, K., et al. 2005, Nature, 434, 1098

Jakobsson, P., Hjorth, J., Fynbo, J. P. U., Watson, D., Pedersen, K., Björnsson, G., \& Gorosabel, J. 2004, ApJ, 617, L21

Kann, D. A., et al. 2010, ApJ, 720, 1513

Kann, D. A., et al. 2008, arXiv:0804.1959

Kocevski, D., et al. 2010, MNRAS, 404, 963

Kouveliotou, C., Meegan, C. A., Fishman, G. J., Bhat, N. P., Briggs, M. S., Koshut, T. M., Paciesas, W. S., \& Pendleton, G. N. 1993, ApJ, 413, L101

La Parola, V., et al. 2006, A\&A, 454, 753

Levan, A. J., Wynn, G. A., Chapman, R., Davies, M. B., King, A. R., Priddey, R. S., \& Tanvir, N. R. 2006a, MNRAS, 368, L1

Levan, A. J., et al. 2006b, ApJ, 648, L9

Levesque, E. M., et al. 2010, MNRAS, 401, 963

Malesani, D., de Ugarte Postigo, A., Levan, A. J., Tanvir, N. R., Hjorth, J., \& D'Avanzo, P. 2009, GRB Coordinates Network, 10156, 1

McBreen, S., et al. 2010, A\&A, 516, 71

Metzger, B. D., Quataert, E., \& Thompson, T. A. 2008, MNRAS, 385, 1455

Nakar, E., Gal-Yam, A., Piran, T., \& Fox, D. B. 2006, ApJ, 640, 849
Narayan, R., Paczynski, B., \& Piran, T. 1992, ApJ, 395, L83

Nysewander, M., Fruchter, A. S., \& Pe'er, A. 2009, ApJ, 701, 824

Paczynski, B. 1991, Acta Astron., 41, 257

Palmer, D. M., et al. 2005, Nature, 434, 1107

Panaitescu, A., Kumar, P., \& Narayan, R. 2001, ApJ, 561, L171

Perley, D. A., Bloom, J. S., Modjaz, M., Miller, A. A., Shiode, J., Brewer, J., Starr, D., \& Kennedy, R. 2008, GRB Coordinates Network, 7889, 1

Perley, D. A., Thoene, C. C., Cooke, J., Bloom, J. S., \& Barton , E. 2007, GRB Coordinates Network, 6739, 1

Perley, D. A., et al. 2009, ApJ, 696, 1871

Perna, R., \& Belczynski, K. 2002, ApJ, 570, 252

Piranomonte, S., et al. 2008, A\&A, 491, 183

Prochaska, J. X., et al. 2006, ApJ, 642, 989

Qin, B., Wu, X., Chu, M., Fang, L., \& Hu, J. 1998, ApJ, 494, L57

Reddy, N. A., \& Steidel, C. C. 2009, ApJ, 692, 778

Roming, P. W. A., et al. 2006, ApJ, 651, 985

Rowlinson, A., et al. 2010a, arXiv:1006.0487

Rowlinson, A., et al. 2010b, MNRAS, submitted

Salvaterra, R., Cerutti, A., Chincarini, G., Colpi, M., Guidorzi, C., \& Romano, P. 2008, MNRAS, 388, L6

Salvaterra, R., Devecchi, B., Colpi, M., \& D'Avanzo, P. 2010, MNRAS, 727

Sari, R., Piran, T., \& Narayan, R. 1998, ApJ, 497, L17

Savaglio, S., Glazebrook, K., \& Le Borgne, D. 2009, ApJ, 691, 182

Soderberg, A. M., et al. 2006, ApJ, 650, 261

Steidel, C. C., Adelberger, K. L., Giavalisco, M., Dickinson, M., \& Pettini, M. 1999, ApJ, 519, 1

Stratta, G., et al. 2007, A\&A, 474, 827

Tanvir, N. R., Chapman, R., Levan, A. J., \& Priddey, R. S. 2005, Nature, 438, 991

Thompson, C., \& Duncan, R. C. 1995, MNRAS, 275, 255

Villasenor, J. S., et al. 2005, Nature, 437, 855

Willmer, C. N. A., et al. 2006, ApJ, 647, 853

Woosley, S. E., \& Bloom, J. S. 2006, ARA\&A, 44, 507 